\title{
On density of subgraphs of Cartesian products
}

\author{
Victor Chepoi, Arnaud Labourel, and Sébastien Ratel \\ Aix Marseille Univ, Université de Toulon, CNRS, LIS, Marseille, France \\ \{victor.chepoi, arnaud.labourel, sebastien.ratel\}@lis-lab.fr
}

\begin{abstract}
In this paper, we extend two classical results about the density of subgraphs of hypercubes to subgraphs $G$ of Cartesian products $G_{1} \square \cdots \square G_{m}$ of arbitrary connected graphs. Namely, we show that $\frac{|E(G)|}{|V(G)|} \leq\left\lceil 2 \max \left\{\operatorname{dens}\left(G_{1}\right), \ldots, \operatorname{dens}\left(G_{m}\right)\right\}\right\rceil \log |V(G)|$, where $\operatorname{dens}(H)$ is the maximum ratio $\frac{\left|E\left(H^{\prime}\right)\right|}{\left|V\left(H^{\prime}\right)\right|}$ taken over all subgraphs $H^{\prime}$ of $H$. We introduce the notions of VC-dimension VC-dim $(G)$ and VC-density VC-dens $(G)$ of a subgraph $G$ of a Cartesian product $G_{1} \square \cdots \square G_{m}$, generalizing the classical Vapnik-Chervonenkis dimension of set-families (viewed as subgraphs of hypercubes). We prove that if $G_{1}, \ldots, G_{m}$ belong to the class $\mathcal{G}(H)$ of all finite connected graphs not containing a given graph $H$ as a minor, then for any subgraph $G$ of $G_{1} \square \cdots \square G_{m}$ the sharper inequality $\frac{|E(G)|}{|V(G)|} \leq \mu(H) \cdot \operatorname{VC}-\operatorname{dim}^{*}(G)$ holds, where $\mu(H)$ is the supremum of the densities of the graphs from $\mathcal{G}(H)$. We refine and sharpen these two results to several specific graph classes. We also derive upper bounds (some of them polylogarithmic) for the size of adjacency labeling schemes of subgraphs of Cartesian products.
\end{abstract}

\section{INTRODUCTION}

A folklore result (see for example [23, Lemma 3.2] and [21]) asserts that if $G=(V, E)$ is an induced $n$-vertex subgraph of the $m$-dimensional hypercube $Q_{m}$, then $\frac{|E|}{|V|} \leq \log n$. This inequality together with the fact that this class of graphs is closed by taking induced subgraphs immediately implies that $n$-vertex subgraphs of hypercubes have $O(\log n)$ density, degeneracy, arbority, and consequently admit $O\left(\log ^{2} n\right)$ adjacency labeling schemes. This density bound $\frac{|E|}{|V|} \leq \log n$ has been refined and sharpened in several directions and these improvements lead to important applications and results.

On the one hand, the edge-isoperimetric problem for hypercubes [8,24 asks for any integer $1 \leq n \leq 2^{m}$ to find an $n$-vertex subgraph $G$ of the $m$-dimensional cube $Q_{m}$ with the smallest edge-boundary $\partial G$, i.e., with the minimum number of edges of $Q_{m}$ running between $G$ and its complement in $Q_{m}$. Since the hypercubes are regular graphs, minimizing the boundary $\partial G$ of $G$ is equivalent to maximizing the number of edges of $G$, and thus to maximizing the density $\frac{|E|}{|V|}$ of $G$. The classical result by Harper [24 nicely characterizes the solutions of the edge-isoperimetric problem for hypercubes: for any $n$, this is the subgraph of $Q_{m}$ induced by the initial segment of length $n$ of the lexicographic numbering of the vertices of $Q_{m}$. One elegant way of proving this result is using the operation of compression [25]. For a generalization of these results and techniques to subgraphs of Cartesian products of other regular graphs and for applications of edge-isoperimetric problems, see the book by Harper [25] and the survey by Bezrukov [8].

On the other hand, to any set family (concept class) $\mathcal{S} \subseteq\{0,1\}^{m}$ with $n$ sets one can associate the subgraph $G(\mathcal{S})=(V, E)$ of the hypercube $Q_{m}$ induced by the vertices corresponding to the sets of $\mathcal{S}$, i.e., $V=\mathcal{S}$. This graph $G(\mathcal{S})$ is called the 1-inclusion graph of $\mathcal{S}$; 1-inclusion graphs have numerous applications in computational learning theory, for example, in prediction strategies 27] and in sample compression schemes [34. Haussler, Littlestone, and Warmuth 27] proposed a prediction strategy for concept classes based on their 1-inclusion graph (called the 1-inclusion prediction strategy) as a natural approach to the prediction model of learning. They provided an upper bound on the worst-case expected risk of the 1-inclusion strategy for a concept class $\mathcal{S}$ by the density of its 1 -inclusion graph $G(\mathcal{S})$ divided by $n$. Moreover, 27, Lemma 2.4] 
establishes a sharp upper bound $\frac{|E|}{|V|} \leq \operatorname{VC}-\operatorname{dim}(\mathcal{S})$ on the density of $G(\mathcal{S})$, where VC-dim $(\mathcal{S})$ is the Vapnik-Chervonenkis dimension of $\mathcal{S}$. While $\mathrm{VC}-\operatorname{dim}(\mathcal{S}) \leq \log n$ always holds, for some concept classes $\mathrm{VC}-\operatorname{dim}(\mathcal{S})$ is much smaller than $\log n$ and thus the inequality $\frac{|E|}{|V|} \leq \mathrm{VC}-\operatorname{dim}(\mathcal{S})$ presents a significant improvement over the folklore inequality $\frac{|E|}{|V|} \leq \log n$. This is the case of maximum concept classes [34 and, more generally, of lopsided systems [7, 9, 35. In this case, $\mathrm{VC}-\operatorname{dim}(\mathcal{S})$ is exactly the dimension of the largest subcube of $G(\mathcal{S})$ and this dimension may not depend at all on the number of sets of $\mathcal{S}$. Haussler 26 presented an elegant proof of the inequality $\frac{|E|}{|V|} \leq \mathrm{VC}-\operatorname{dim}(\mathcal{S})$ using the shifting (push-down) operation (which can be compared with the compression operation for edge-isoperimetric problem). He used this density result to give an upper bound on the $\epsilon$-packing number (the maximum number of disjoint balls of a radius $\epsilon$ of $Q_{m}$ with centers at the vertices of $G(\mathcal{S})$ ), and this result can be viewed as a far-reaching generalization of the classical Sauer lemma [41]. The inequality of Haussler et al. 27] as well as the classical notions of VC-dimension and the Sauer lemma have been subsequently extended to the subgraphs of Hamming graphs, i.e., from binary alphabets to arbitrary alphabets; see [28,37,39]. Cesa-Bianchi and Haussler [14] presented a graph-theoretical generalization of the Sauer Lemma for the $m$-fold $F^{m}=F \square \cdots \square F$ Cartesian products of arbitrary undirected graphs $F$.

In this paper, we extend the inequalities $\frac{|E|}{|V|} \leq \log n$ and $\frac{|E|}{|V|} \leq \operatorname{VC}-\operatorname{dim}(\mathcal{S})$ to $n$-vertex subgraphs $G=(V, E)$ of Cartesian products $\Gamma:=G_{1} \square \cdots \square G_{m}$ of arbitrary connected graphs $G_{1}, \ldots, G_{m}$. Namely, in Theorem 2, we show that the density of $G$ is at most $2 \log n$ times the largest density of a factor of $\Gamma$. To extend the density result of [27], we define the notions of (minor and induced) VC-dimensions and VC-densities of subgraphs $G$ of arbitrary Cartesian products and show that if all factors $G_{1}, \ldots, G_{m}$ do not contain a fixed subgraph $H$ as a minor, then the density of any subgraph $G=(V, E)$ of $\Gamma$ is at most $\mu(H)$ times the VC-dimension VC-dim* $(G)$ of $G: \frac{|E|}{|V|} \leq \mu(H) \cdot \operatorname{VC}-\operatorname{dim}^{*}(G)$, where $\mu(H)$ is a constant such that any graph not containing $H$ as a minor has density at most $\mu(H)$ (it is well known 18] that if $r:=|V(H)|$, then $\mu(H) \leq c r \sqrt{\log r}$ for a universal constant $c)$. We conjecture that in fact $\frac{|E|}{|V|} \leq \mathrm{VC}-\operatorname{dens}^{*}(G)$ holds, where VC-dens* $(G)$ is the VC-density of $G$. We consider several classes of graphs for which sharper inequalities hold. Since by Nash-Williams's theorem [36] all such inequalities provide upper bounds for arboricity and since by a result of Kannan, Naor, and Rudich 32 bounded arboricity implies bounded adjacency labeling schemes, in the last section of the paper we present the applications of our results to the design of compact adjacency labeling schemes for subgraphs of Cartesian products (which was one of our initial motivations).

The canonical metric representation theorem of Graham and Winkler [22] asserts that any connected finite graph $G$ has a unique isometric embedding into the Cartesian product $\Pi_{i=1}^{m} G_{i}$ in which each factor $G_{i}$ is prime (i.e., not further decomposable this way) and this representation can be computed efficiently; for proofs and algorithms, see the books 17,23. Thus our results have a general nature and show that it suffices to bound the density of prime graphs. For many classes of graphs occurring in metric graph theory [6], the prime graphs have special structure. For example, the primes for isometric subgraphs of hypercubes (which have been characterized in a nice way by Djoković [19]) are the $K_{2}$. Thus the density of isometric subgraphs of hypercubes (and more generally, of subgraphs of hypercubes) is upper bounded by their VC-dimension. Shpectorov [42] proved that the primes of graphs which admit a scale embedding into a hypercube are exactly the subgraphs of octahedra and isometric subgraphs of halved cubes. In Section 7, we will show how to bound the density of subgraphs of Cartesian products of octahedra. In another paper [16] we will define an appropriate notion of VC-dimension for subgraphs of halved cubes and we will use it to upper bound the density of such graphs. The papers [10] and [11] investigate the local-to-global structure of graphs which are retracts of Cartesian products of chordal graphs, 
bridged and weakly bridged graphs, respectively (bridged graphs are the graphs in which all isometric cycles have length 3). 2-Connected chordal graphs and bridged or weakly bridged graphs are prime. Notice also that the bridged and weakly bridged graphs are dismantlable (see, for example, [11, Section 7]). In Section 7, we present sharper density inequalities for subgraphs of Cartesian products of chordal graphs and of dismantlable graphs, which can be directly applied to the classes of graphs from [10] and [11]. For other such classes of graphs occurring in metric graph theory, see the survey [6] and the papers [12,13].

\section{Preliminaries}

In this section, we define the basic notions and concepts used throughout the paper. Some specific notions (some classes of graphs, adjacency labeling schemes, etc) will be introduced when appropriate.

\subsection{Basic definitions.}

2.1.1. Density. All graphs $G=(V, E)$ occurring in this note are finite, undirected, and simple. The closed neighbourhood of a vertex $v$ is denoted by $N[v]$ and consists of $v$ and the vertices adjacent to $v$. The degree $d(v)$ of a vertex $v$ is the number of edges of $G$ incident to $v$. The number $d(G)=\frac{1}{|V|} \sum_{v \in G} d(v)=\frac{2|E|}{|V|}$ is the average degree of $G$.

The maximum average degree $\operatorname{mad}(G)$ of $G$ is the maximum average degree of a subgraph $G^{\prime}$ of $G: \operatorname{mad}(G)=\max \left\{d\left(G^{\prime}\right): G^{\prime}\right.$ is a subgraph of $\left.G\right\}$.

The density dens $(G)$ of $G$ will be the maximal ratio $\left|E\left(G^{\prime}\right)\right| /\left|V\left(G^{\prime}\right)\right|$ over all its subgraphs $G^{\prime}$.

Density and maximum average degree are closely related, namely $\operatorname{dens}(G)=\frac{\operatorname{mad}(G)}{2}=$ $\max \left\{\frac{d\left(G^{\prime}\right)}{2}: G^{\prime} \subseteq G\right\}$ holds, but they quantify different aspects of $G$. We will use both numbers, depending on the circumstances: $\operatorname{dens}(G)$ will be used to express a global parameter of $G$ in a result, and $\operatorname{mad}(G)$ will be used in proofs when we have to look at a local parameter (degrees) of $G$. We will use the following simple observation:

Lemma 1. Let $G$ be a simple and connected graph. Then $G$ has two vertices of degree at most $\lceil\operatorname{mad}(G)\rceil$.

Proof. Let $\mu(G):=\lceil\operatorname{mad}(G)\rceil$ and $n$ the number of vertices of $G$. Assume for contradiction that there exists connected graph $G$ with one vertex $v_{0}$ of degree at most $\mu(G)$ and $n-1$ vertices of degree at least $\mu(G)+1$. Since $G$ is connected, $d\left(v_{0}\right) \geq 1$ and we obtain $\operatorname{mad}(G) \geq d(G) \geq$ $\frac{(\mu(G)+1)(n-1)+1}{n}=\mu(G)+1-\frac{\mu(G)}{n}>\mu(G)$, leading to a contradiction.

2.1.2. Cartesian products [17, 23]. Let $G_{1}, \ldots, G_{m}$ be a family of $m$ connected graphs. The Cartesian product $\Gamma:=\prod_{i=1}^{m} G_{i}=G_{1} \square \cdots \square G_{m}$ is a graph defined on the set of all $m$-tuples $\left(x_{1}, \ldots, x_{m}\right), x_{i} \in V\left(G_{i}\right)$, where two vertices $x=\left(x_{1}, \ldots, x_{m}\right)$ and $y=\left(y_{1}, \ldots, y_{m}\right)$ are adjacent if and only if there exists an index $1 \leq j \leq m$ such that $x_{j} y_{j} \in E\left(G_{j}\right)$ and $x_{i}=y_{i}$ for all $i \neq j$. If $u v$ is an edge of the factor $G_{i}$, then all edges of $\Gamma$ running between two vertices of the form $\left(v_{1}, \ldots, v_{i-1}, u, v_{i+1}, \ldots, v_{m}\right)$ and $\left(v_{1}, \ldots, v_{i-1}, v, v_{i+1}, \ldots, v_{m}\right)$ will be called edges of type uv. A factor $G_{i}$ is called a non-trivial factor of $\Gamma$ if $G_{i}$ contains at least two vertices. A graph $G$ is prime if it can not be represented as a Cartesian product of two non-trivial graphs.

The $m$-dimensional hypercube $Q_{m}$ is the Cartesian product of $m$ copies of $K_{2}$ with $V\left(K_{2}\right)=$ $\{0,1\}$, i.e., $Q_{m}=K_{2} \square \cdots \square K_{2}$. Equivalently, $Q_{m}$ has the subsets $S$ of a set $X$ of size $m$ as the vertex-set and two such sets $A$ and $B$ are adjacent in $Q_{m}$ if and only if $|A \Delta B|=1$.

A subproduct $\Gamma^{\prime}$ of a Cartesian product $\Gamma=\prod_{i=1}^{m} G_{i}$ is a product such that $\Gamma^{\prime}=\prod_{j=1}^{k} G_{i_{j}}^{\prime}$, where each $G_{i_{j}}^{\prime}$ is a connected non-trivial subgraph of $G_{i_{j}}$. A subproduct $\Gamma^{\prime}=\prod_{j=1}^{k} G_{i_{j}}^{\prime}$ in which each factor $G_{i_{j}}^{\prime}$ is an edge of $G_{i_{j}}$ is called a cube-subproduct of $\Gamma$. 

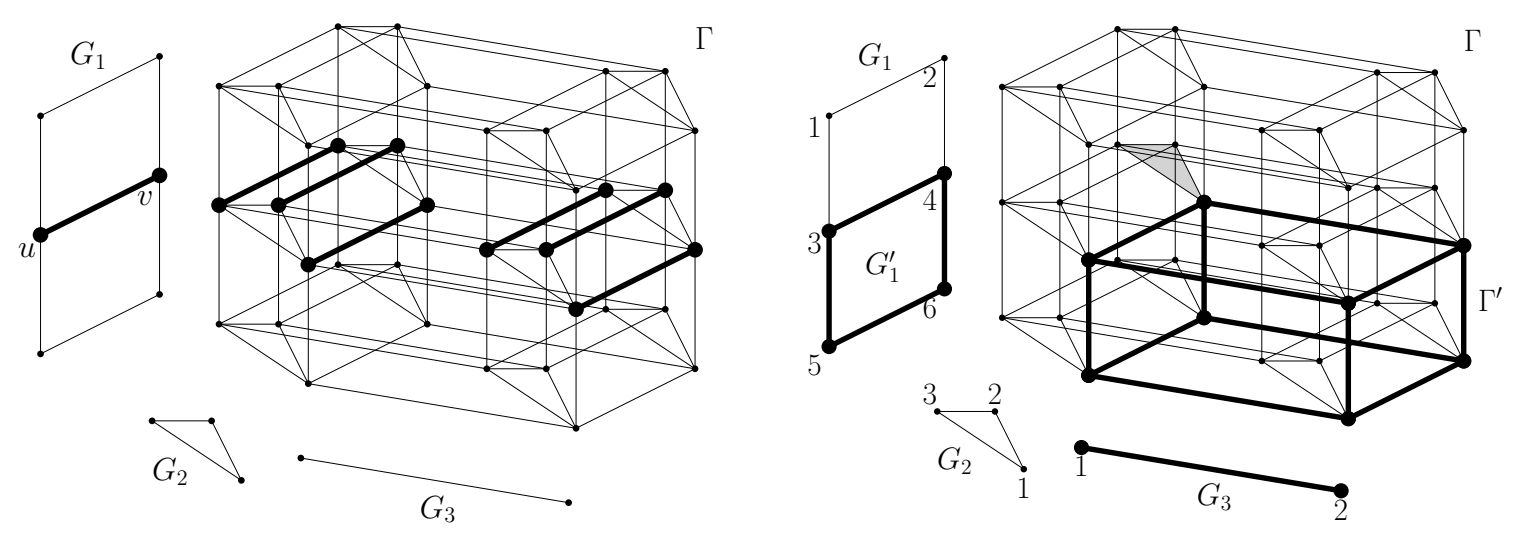

Figure 1. On left, an example of a Cartesian product $\Gamma:=G_{1} \square G_{2} \square G_{3}$ and an edge $u v \in E\left(G_{1}\right)$ and its copies in $\Gamma$ (the edges of type $u v$ of $\Gamma$ ). On right, a subproduct (which is also a cube-subproduct) $\Gamma^{\prime}:=G_{1}^{\prime} \square G_{3}^{\prime}$ of $\Gamma$ and the fiber (in gray) $F((4,1)):=\{(4,1,1),(4,2,1),(4,3,1)\}$ of the vertex $v=(4,1)$ of $\Gamma^{\prime}$.

Given a vertex $v^{\prime}=\left(v_{i_{1}}^{\prime}, \ldots, v_{i_{k}}^{\prime}\right)$ of $\Gamma^{\prime}$, we say that a vertex $v=\left(v_{1}, \ldots, v_{m}\right)$ of $\Gamma$ is an extension of $v^{\prime}$ if $v_{i_{j}}=v_{i_{j}}^{\prime}$ for all $j \in\{1, \ldots, k\}$. We denote by $F\left(v^{\prime}\right)$ the set of all extensions $v$ in $\Gamma$ of a vertex $v^{\prime}$ of a subproduct $\prod_{j=1}^{k} G_{i_{j}}^{\prime}$ and call $F\left(v^{\prime}\right)$ the fiber of $v^{\prime}$ in the product $\Gamma$ (see Figure 1 for an illustration of these notions).

Let $G$ be a subgraph of a Cartesian product $\Gamma$ and $\Gamma^{\prime}$ be a subproduct of $\Gamma$. The trace of $V(G)$ on $V\left(\Gamma^{\prime}\right)$ consists of all vertices $v^{\prime}$ of $\Gamma^{\prime}$ such that $F\left(v^{\prime}\right) \cap V(G) \neq \emptyset$. The projection of $G$ on $\Gamma^{\prime}$ is the subgraph $\pi_{\Gamma^{\prime}}(G)$ of $\Gamma^{\prime}$ induced by the trace of $V(G)$ on $V\left(\Gamma^{\prime}\right)$. We will denote the projection of $G$ on the $i$ th factor $G_{i}$ of $\Gamma$ by $\pi_{i}(G)$ instead of $\pi_{G_{i}}(G)$.

The following lemma must be well-known, but we have not found it in the literature. Its proof was communicated to us by François Dross (which we would like to acknowledge).

Lemma 2. If $\Gamma=\prod_{i=1}^{m} G_{i}$, then $\operatorname{dens}(\Gamma)=\sum_{i=1}^{m} \operatorname{dens}\left(G_{i}\right)$, i.e., if $G_{i}^{\prime}=\left(V_{i}^{\prime}, E_{i}^{\prime}\right)$ is a densest subgraph of $G_{i}=\left(V_{i}, E_{i}\right)$, then $\Gamma^{\prime}=\prod_{i=1}^{m} G_{i}^{\prime}$ is a densest subgraph of $\Gamma$.

Proof. The graph $\Gamma^{\prime}$ has $\left|V_{1}^{\prime}\right| \cdot \ldots \cdot\left|V_{m}^{\prime}\right|$ vertices and $\sum_{i=1}^{m}\left|E_{i}^{\prime}\right| \cdot\left(\prod_{j=1, j \neq i}^{m}\left|V_{j}^{\prime}\right|\right)$ edges. Therefore $\frac{\left|E^{\prime}\right|}{\left|V^{\prime}\right|}=\sum_{i=1}^{m} \frac{\left|E_{i}^{\prime}\right|}{\left|V_{i}^{\prime}\right|}=\sum_{i=1}^{m} \operatorname{dens}\left(G_{i}\right)$, showing that $\operatorname{dens}(\Gamma) \geq \sum_{i=1}^{m} \operatorname{dens}\left(G_{i}\right)$.

We will prove now the converse inequality $\operatorname{dens}(\Gamma) \leq \sum_{i=1}^{m} \operatorname{dens}\left(G_{i}\right)$. Since the Cartesian product operation is associative, it suffices to prove this inequality for two factors. Let $\Gamma=$ $G_{1} \square G_{2}$ and let $G=(V, E)$ be a densest subgraph of $\Gamma$. We will call the edges of $G$ arising from $G_{1}$ horizontal edges and those arising from $G_{2}$ vertical edges and denote the two edge-sets by $E_{h}$ and $E_{v}$. Then $\frac{|E|}{|V|}=\frac{\left|E_{h}\right|}{|V|}+\frac{\left|E_{v}\right|}{|V|}$. Thus it suffices to prove the inequalities $\frac{\left|E_{h}\right|}{|V|} \leq \operatorname{dens}\left(G_{1}\right)$ and $\frac{\left|E_{v}\right|}{|V|} \leq \operatorname{dens}\left(G_{2}\right)$. First, we will establish the first inequality. For each vertex $x \in V_{2}$ we will denote by $L(x)=(V(x), E(x))$ the subgraph of $G$ induced by the vertices of $G$ having $x$ as their second coordinate (this subgraph is called the $x$-layer of $G$, see Fig. 2(a) for an illustration). All horizontal edges of $G$ and all vertices of $G$ are distributed in such layers and the layers are pairwise disjoint. Each layer $L(x)$ is isomorphic to a subgraph of $G_{1}$, thus $\operatorname{dens}(L(x)) \leq \operatorname{dens}\left(G_{1}\right)$ for any $x \in V_{2}$. Therefore,

$$
\frac{\left|E_{h}\right|}{|V|}=\frac{\sum_{x \in V_{2}}|E(x)|}{\sum_{x \in V_{2}}|V(x)|} \leq \max _{x \in V_{2}}\left\{\frac{|E(x)|}{|V(x)|}\right\} \leq \operatorname{dens}\left(G_{1}\right),
$$




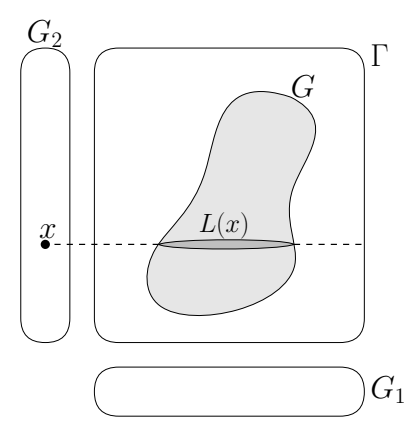

(a)

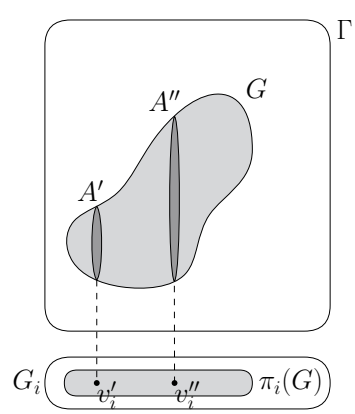

(b)

Figure 2. To the proofs of Lemma 2 and Theorem 2 .

as required (we used the inequality $\frac{\sum_{i=1}^{m} a_{i}}{\sum_{i=1}^{m} b_{i}} \leq \max \left\{\frac{a_{i}}{b_{i}}\right\}$ for nonnegative numbers $a_{1}, \ldots, a_{m}$ and $\left.b_{1}, \ldots, b_{m}\right)$. The second inequality can be shown with a similar argument.

2.1.3. Minors [18]. A minor of a graph $G$ is a graph $M$ obtained from a subgraph $G^{\prime}$ of $G$ by contracting some edges. Equivalently, $M$ is a minor of a connected graph $G$ if there exists a partition of vertices of $G$ into connected subgraphs $\mathcal{P}=\left\{P_{1}, \ldots, P_{t}\right\}$ and a bijection $f: V(M) \rightarrow$ $\mathcal{P}$ such that if $u v \in E(M)$ then there exists an edge of $G$ running between the subgraphs $f(u)$ and $f(v)$ of $\mathcal{P}$, i.e., after contracting each subgraph $P_{i} \in \mathcal{P}$ into a single vertex we will obtain a graph containing $M$ as a spanning subgraph. A class of graphs $\mathcal{G}$ is called minor-closed if for any graph $G$ from $\mathcal{G}$ all minors of $G$ also belong to $\mathcal{G}$.

A minor-subproduct of a Cartesian product $\Gamma:=\prod_{i=1}^{m} G_{i}$ of connected graphs is a Cartesian product $M:=\prod_{i=1}^{m} M_{i}$, where $M_{i}$ is a minor of $G_{i}$ for all $1 \leq i \leq m$. Let $\mathcal{P}_{i}=\left\{P_{1}^{i}, \ldots, P_{t_{i}}^{i}\right\}$ denote the partition of $G_{i}$ defining the minor $M_{i}$ and let $\mathcal{P}:=\mathcal{P}_{1} \times \cdots \times \mathcal{P}_{m}$. Notice that $\mathcal{P}$ is a partition of the vertex set of the Cartesian product $\prod_{i=1}^{m} G_{i}$.

2.2. VC-dimension and VC-density. Let $\mathcal{S}$ be a family of subsets of a finite set $X=$ $\left\{e_{1}, \ldots, e_{m}\right\}$, i.e., $\mathcal{S} \subseteq 2^{X}$. $\mathcal{S}$ can be viewed as a subset of vertices of the $m$-dimensional hypercube $Q_{m}$. Denote by $G(\mathcal{S})$ the subgraph of $Q_{m}$ induced by the vertices of $Q_{m}$ corresponding to the sets of $\mathcal{S} ; G(\mathcal{S})$ is also called the 1-inclusion graph of $\mathcal{S}$ 26, 27) (in [20, such graphs were called cubical graphs). Vice-versa, any induced subgraph $G=(V, E)$ of the hypercube $Q_{m}$ corresponds to a family of subsets $\mathcal{S}(G)$ of $2^{X}$ with $|X|=m$ such that $G$ is the 1-inclusion graph of $\mathcal{S}(G)$.

A subset $Y$ of $X$ is said to be shattered by $\mathcal{S}$ if for any $Y^{\prime} \subseteq Y$, there exists a set $S \in \mathcal{S}$ such that $S \cap Y=Y^{\prime}$. The Vapnik-Chervonenkis's dimension [31,43] VC- $\operatorname{dim}(\mathcal{S})$ of $\mathcal{S}$ is the cardinality of the largest subset of $X$ shattered by $\mathcal{S}$. Viewing $Q_{m}$ as the $m$-fold Cartesian product $K_{2} \square \cdots \square K_{2}$, the shattering operation can be redefined in more graph-theoretical terms as follows. For $Y \subseteq X$ denote by $\Gamma_{Y}$ the Cartesian product of the factors of $Q_{m}$ indexed by $e_{i} \in Y\left(\Gamma_{Y}\right.$ is a $|Y|$-dimensional cube). Then a set $Y \subseteq X$ is shattered by $\mathcal{S}$ (or by $G(\mathcal{S})$ ) if $\pi_{\Gamma_{Y}}(G(\mathcal{S}))=\Gamma_{Y}$; see Fig. 4, 5, and 6 for an illustration.

We continue with our main definitions of VC-dimension and VC-density for subgraphs of Cartesian products of connected graphs. First, we define these notions with respect to subproducts.

Definition 1. A subproduct $\Gamma^{\prime}:=\prod_{j=1}^{k} G_{i_{j}}^{\prime}$ of a Cartesian product $\Gamma=\prod_{i=1}^{m} G_{i}$ is shattered by $G$ if $F\left(v^{\prime}\right) \cap V(G) \neq \emptyset$ for any vertex $v^{\prime}$ of $\Gamma^{\prime}$, i.e., if $\pi_{\Gamma^{\prime}}(G)=\Gamma^{\prime}$. The (induced) VC-dimension VC-dim $(G)$ of $G$ with respect to the Cartesian product $\Gamma$ is the largest number of non-trivial 
factors in a subproduct $\Gamma^{\prime}$ of $\Gamma$ shattered by $G$. Equivalently, VC-dim $(G)$ is the largest dimension of a cube-subproduct of $\Gamma$ shattered by $G$ (since each factor of $\Gamma^{\prime}$ is non-trivial). The $V C$-density VC-dens $(G)$ of $G$ is the largest density of a subproduct $\Gamma^{\prime}$ shattered by $G$.

In the same vein, we consider now the notion of the VC-dimension and VC-density of subgraphs $G$ of a Cartesian product $\Gamma$ with respect to minor subproducts.

Definition 2. Let $M:=\prod_{i=1}^{m} M_{i}$ be a minor subproduct of $\Gamma=\prod_{i=1}^{m} G_{i}$. Let $\mathcal{P}:=\mathcal{P}_{1} \times \cdots \times \mathcal{P}_{m}$ be the partition of $\Gamma$ such that $\mathcal{P}_{i}=\left\{P_{1}^{i}, \ldots, P_{t_{i}}^{i}\right\}$ is the partition of $G_{i}$ defining the minor $M_{i}$, $i=1, \ldots, m$. The minor-subproduct $M$ is shattered by $G$ if any set $P_{l_{1}}^{1} \times \cdots \times P_{l_{m}}^{m}$ of $\mathcal{P}$ (where $l_{1}=1, \ldots, t_{1}, \ldots$, and $\left.l_{m}=1, \ldots, t_{m}\right)$ contains a vertex of $G$. The (minor) $V C$-dimension VC-dim* $(G)$ of $G$ with respect to $\Gamma$ is the largest number of non-trivial factors of a minorsubproduct $M$ of $\Gamma$ shattered by $G$. The (minor) $V C$-density VC-dens* $(G)$ of $G$ is the largest density $\operatorname{dens}(M)$ of a minor subproduct $M$ shattered by $G$.

Example 1. In Fig. 3(1), we present a subgraph $G$ of the Cartesian product $\Gamma=G_{1} \square G_{2}$ of two graphs $G_{1}$ and $G_{2}$. In Fig. 3(2), we provide a partition of $V(\Gamma)$ which induces a shattered minor isomorphic to the square $K_{2} \square K_{2}$. On the other hand, in Fig. 3(3) we present a partition of $\Gamma$ which induces the Cartesian product $P_{3} \square P_{2}$ of the paths of length 2 and length 1 (see Fig. 3(4)) and this product is not shattered by $G$.

Example 2. The inequalities VC-dim $(G) \leq \mathrm{VC}-\operatorname{dim}^{*}(G)$ and VC-dens $(G) \leq \mathrm{VC}-\operatorname{dens}{ }^{*}(G)$ hold for any subgraph $G$ of $\Gamma$. In Fig.7, we present a simple example of a subgraph $G$ of the product of two 2-paths for which VC-dim $(G)=1$ and VC-dim* $(G)=2$.

Remark 1. In case of $m$-fold Cartesian products $F^{m}$ of a fixed graph $F$, Cesa-Bianchi and Haussler [14] defined the notion of a $d$-dimensional projected cube, which in this case coincides with our notion of shattered cube-subfactor.

Remark 2. As in the case of the classical VC-dimension, VC-dim $(G)$ and VC-dim* $(G)$ are defined with respect to an embedding of $G$ as a subgraph of the Cartesian product $\Pi_{i=1}^{m} G_{i}$. For example, if $G$ is the path $P_{5}$ (with 5 vertices and 4 edges) and this path is embedded in the 4-cube $Q_{4}$ such that the end-vertices of $P_{5}$ are $(0,0,0,0)$ and $(1,1,1,1)$, then $\mathrm{VC}$-dim* $\left(P_{5}\right)=$ $\mathrm{VC}-\operatorname{dim}\left(P_{5}\right)=1$. However, if $P_{5}$ is embedded in $Q_{3}$ (which can be viewed as a face of $Q_{4}$ ) such the end-vertices of $P_{5}$ are $(0,0,0)$ and $(1,1,0)$, then $\operatorname{VC}-\operatorname{dim}^{*}\left(P_{5}\right)=\operatorname{VC}-\operatorname{dim}\left(P_{5}\right)=2$. For illustration of the two embeddings of $P_{5}$ see Figures 5 and 6 .

In this paper, when we speak about VC-dim $(G)$ or VC-dim* $(G)$ we assume that an embedding of $G$ as an induced subgraph of a Cartesian product $G_{1} \square \cdots \square G_{m}$ is given. This is also essential from the computational point of view because already recognizing if a graph $G$ is a subgraph of a hypercube is NP-complete [2].

Remark 3. We present some motivation for the names "VC-dimension" and "VC-density" in the general setting of subgraphs of Cartesian products and for the way these concepts have been defined. First notice that in case of subgraphs $G$ of hypercubes $Q_{m}$, i.e., set-systems, the equality $\mathrm{VC}-\operatorname{dim}(G)=\mathrm{VC}-\operatorname{dim}^{*}(G)=\mathrm{VC}-\operatorname{dens}(G)=\mathrm{VC}-\operatorname{dens}{ }^{*}(G)=\mathrm{VC}-\operatorname{dim}(\mathcal{S}(G))$ holds and these numbers coincide with the dimension of the largest subcube $Q$ of $Q_{m}$ shattered by $G$. This is because for hypercubes, i.e., Cartesian products of $K_{2}$, the notions of subproducts, cube-subproducts, and minor-subproducts coincide. All these dimensions coincide with the degrees of the vertices of $Q$ and thus coincide with the average degree $\operatorname{mad}(Q)$ of $Q$. This is one explanation why the VC-density of subgraphs of Cartesian products has been defined via the average degrees of shattered subproducts or shattered minor subproducts. Second, Lemma 2 shows that subproducts are densest subgraphs of Cartesian products. Therefore, one can expect 


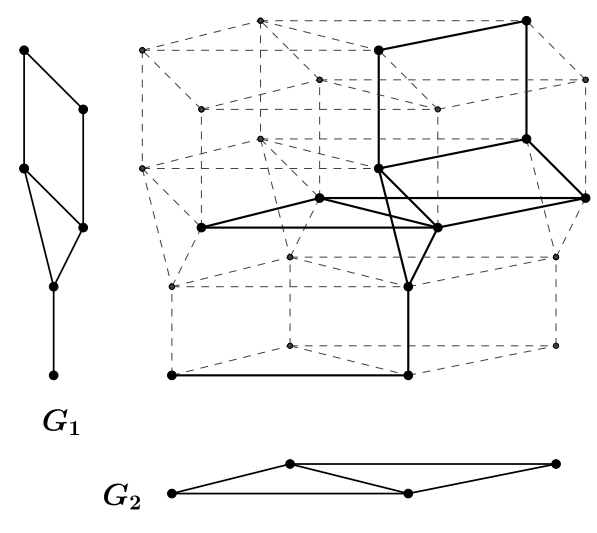

$(1)$

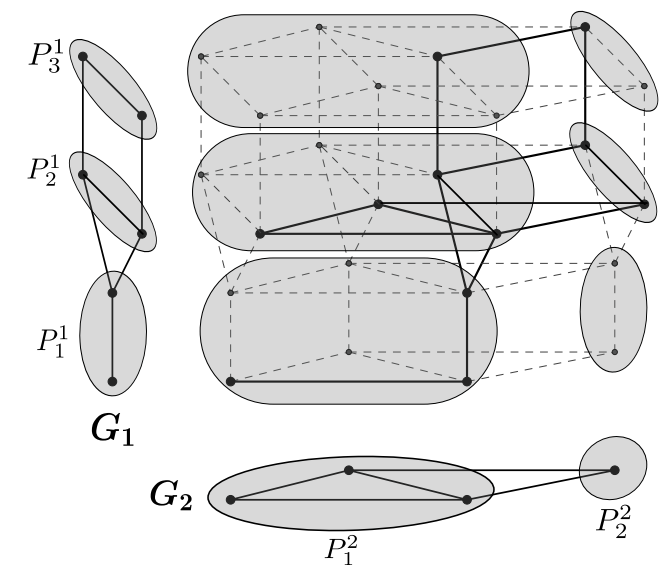

(3)

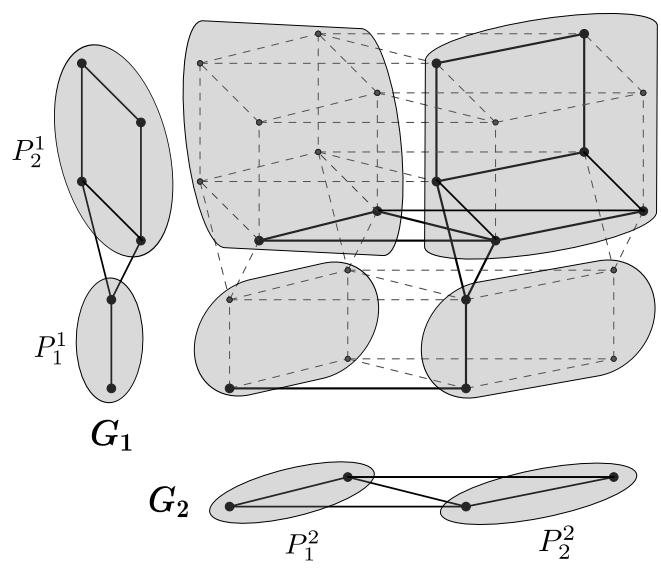

(2)

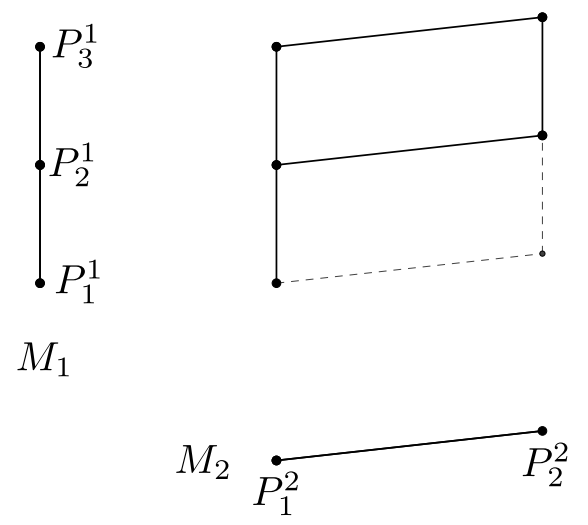

(4)

Figure 3. A subgraph $G$ of a Cartesian product of $\Gamma=G_{1} \square G_{2}$ and two partitions of $V(\Gamma)$, first inducing a shattered by $G$ minor-subproduct and second inducing a subproduct not shattered by $G$.

that in case of subgraphs $G$ of Cartesian products, the densest subproducts shattered by $G$ provide an upper bound for the density of $G$. Third, for subgraphs of general products one cannot define VC-dens $(G)$ or VC-dens* $(G)$ as just the maximum number of factors in a shattered (minor) subproduct because the factors in this subproduct may have completely different numbers of vertices, edges, or average degrees. Finally, we use "VC" in the names because it concerns shattering, closely related to classical VC-dimension.

\section{Related WORK}

3.1. Subgraphs of hypercubes. In this subsection, we briefly review the inductive method of bounding the density of subgraphs $G$ of hypercubes by the VC-dimension $d$ of $\mathcal{S}=\mathcal{S}(G)$ :

Theorem 1. [27, Lemma 2.4] $\frac{|E(G)|}{|V(G)|} \leq d$.

There are several ways to prove this result (see [26] for a proof using shifting operations, or [8,24 for edge-isoperimetric inequalities method), but our proofs in Section 6 use the same idea as the inductive method we recall now. A similar proof also applies to the classical Sauer lemma asserting that sets families of $\{0,1\}^{m}$ of VC-dimension $d$ have size $O\left(m^{d}\right)$ (generalizations 


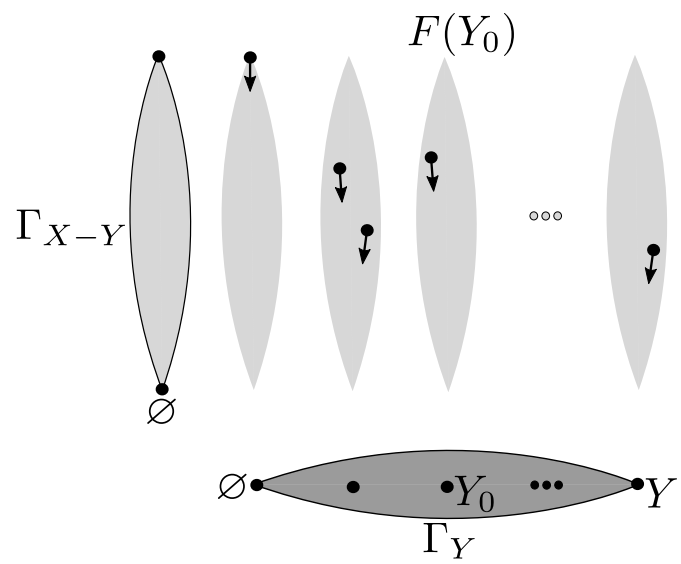

Figure 4. A set $Y$ shattered by a set family $\mathcal{S}$ (the sets of $\mathcal{S}$ are represented by black vertices in the gray fibers, see $F\left(Y_{0}\right)$, for example).

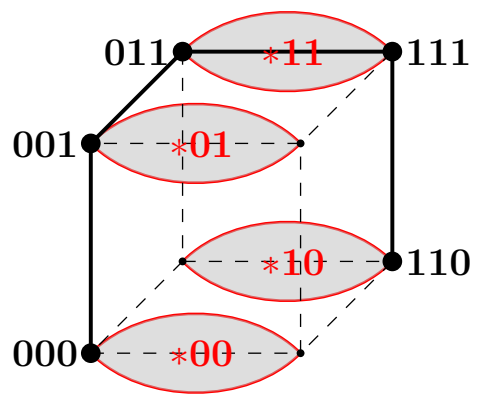

FiguRE 6. An embedding of $P_{5}$ in $Q_{3}$ with VC-dimension 2 $(\{000,010,001,011\}$ is shattered).

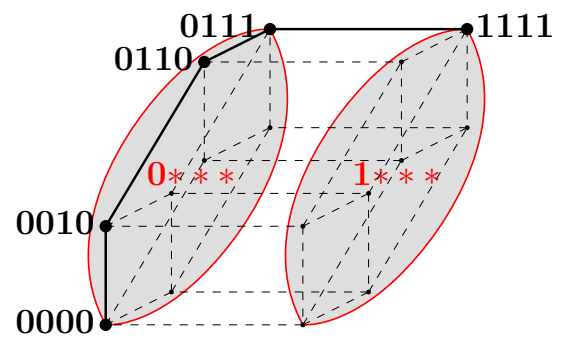

Figure 5. An embedding of $P_{5}$ in $Q_{4}$ with VC-dimension 1 ( $\{0000,1000\}$ is shattered).

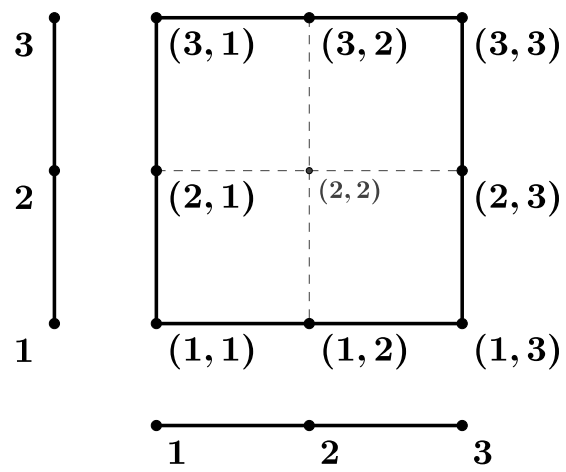

FiguRE 7. A subgraph $G$ of $P_{3} \square P_{3}$ that does not shatter any subproduct with 2 factors but that shatters a minorsubproduct induced by $\mathcal{P}=$ $\{\{1,2\},\{3\}\} \times\{\{1\},\{2,3\}\}$.

of Theorem 1 and of the Sauer lemma were provided for subgraphs of Hamming graphs in [28,40]). Both proofs are based on the following fundamental lemma. For a finite set $X$ and $e \in X$, let $\mathcal{S}_{e}=$ $\left\{S^{\prime} \subseteq X \backslash\{e\}: S^{\prime}=S \cap X\right.$ for some $\left.S \in \mathcal{S}\right\}$ and $\mathcal{S}^{e}=\left\{S^{\prime} \subseteq X \backslash\{e\}: S^{\prime}\right.$ and $S^{\prime} \cup\{e\}$ belong to $\left.\mathcal{S}\right\}$.

Lemma 3. $|\mathcal{S}|=\left|\mathcal{S}_{e}\right|+\left|\mathcal{S}^{e}\right|, \mathrm{VC}-\operatorname{dim}\left(\mathcal{S}_{e}\right) \leq d$, and $\mathrm{VC}-\operatorname{dim}\left(\mathcal{S}^{e}\right) \leq d-1$.

The proof of the inequality $|E(G)| \leq d \cdot|V(G)|$ in Theorem 1 provided by Haussler, Littlestone, and Warmuth 27] is by induction using Lemma 3. For $e \in X$, denote by $G_{e}$ and $G^{e}$ the subgraphs of $2^{X-\{e\}}$ induced by $\mathcal{S}_{e}$ and $\mathcal{S}^{e}$. Then $\left|E\left(G_{e}\right)\right| \leq d\left|V\left(G_{e}\right)\right|=d\left|\mathcal{S}_{e}\right|$ and $E\left(G^{e}\right) \leq(d-1)\left|V\left(G^{e}\right)\right|=$ $(d-1)\left|\mathcal{S}^{e}\right|$ by Lemma 3 and induction hypothesis. The graph $G_{e}$ is obtained from $G$ by contracting the set $F$ of edges of type $e$ of $G$. The vertex set of $G^{e}$ is in bijection with $F$ and two vertices of $G^{e}$ are adjacent iff the corresponding edges of $F$ belong to a common square. By Lemma 3. $|V(G)|=|\mathcal{S}|=\left|\mathcal{S}_{e}\right|+\left|\mathcal{S}^{e}\right|=\left|V\left(G_{e}\right)\right|+\left|V\left(G^{e}\right)\right|$. The edges of $G$ which lead to loops or 
multiple edges of $G_{e}$ are the edges of $G^{e}$ and of $F$, hence $|E(G)| \leq\left|E\left(G_{e}\right)\right|+\left|E\left(G^{e}\right)\right|+|F|=$ $\left|E\left(G_{e}\right)\right|+\left|E\left(G^{e}\right)\right|+\left|V\left(G^{e}\right)\right|$. From this (in)equality and after some calculation, one deduce that $\frac{|E(G)|}{|V(G)|} \leq d$.

3.2. Other notions of VC-dimension in graphs. We continue with a brief survey of other existing notions of VC-dimension in graphs. Haussler and Welzl [29] defined the VC-dimension of a graph $G=(V, E)$ as the VC-dimension of the set family of closed neighborhoods of vertices of $G$. It was shown in [29] that this VC-dimension of planar graphs is at most 4. Anthony, Brightwell, and Cooper [5] proved that this VC-dimension is at most $d$ if $G$ does not contain $K_{d+1}$ as a minor (they also investigated this notion of VC-dimension for random graphs). These two results have been extended in [15, Proposition 1 \& Remark 1] to the families of closed balls of any fixed radius. Kranakis et al. [33] considered the VC-dimension for other natural families of sets of a graph: the families induced by trees, connected subgraphs, paths, cliques, stars, etc.). They investigated the complexity issues for computing these VC-dimensions and for some of them they presented upper bounds in terms of other graph-parameters. The concept of VCdimension of the family of shortest paths (in graphs with unique shortest paths) was exploited in [1] to improve the time bounds of query algorithms for point-to-point shortest path problems in real world networks (in particular, for road networks).

\section{OUR RESUlts}

The main purpose of this paper is to generalize the density results about subgraphs of hypercubes to subgraphs $G$ of Cartesian products of connected graphs $G_{1}, \ldots, G_{m}$. Namely, we will prove the following two results:

Theorem 2. Let $G$ be a subgraph of a Cartesian product $G_{1} \square \cdots \square G_{m}$ of connected graphs $G_{1}, \ldots, G_{m}$ and let $\beta \quad:=\left\lceil\max \left\{\operatorname{mad}\left(G_{1}\right), \ldots, \operatorname{mad}\left(G_{m}\right)\right\}\right\rceil, \quad \beta_{0}=$ $\left\lceil\max \left\{\operatorname{mad}\left(\pi_{1}(G)\right), \ldots, \operatorname{mad}\left(\pi_{m}(G)\right)\right\}\right\rceil$. Then

$$
\frac{|E(G)|}{|V(G)|} \leq \beta_{0} \log |V(G)| \leq \beta \log |V(G)| .
$$

For a graph $H$, denote by $\mathcal{G}(H)$ the set of all finite $H$-minor-free graphs, i.e., graphs not having $H$ as a minor. By results of Mader, Kostochka, and Thomason (see [18, Chapter 8.2]), any graph $G$ with average degree $d(G) \geq c r \sqrt{\log r}$ has the complete graph $K_{r}$ on $r$ vertices as a minor. Therefore, for each graph $H$ on $r$ vertices, there exists a constant $\mu(H) \leq c r \sqrt{\log r}$ such that all graphs $G$ from $\mathcal{G}(H)$ have average degree $d(G) \leq \mu(H)$. In case when the factors of the product $\Gamma$ belong to $\mathcal{G}(H)$ the following result, generalizing Theorem 1 and sharpening Theorem 2, holds:

Theorem 3. Let $H$ be a graph and let $G$ be a subgraph of a Cartesian product $\Gamma=G_{1} \square \cdots \square G_{m}$ of connected graphs $G_{1}, \ldots, G_{m}$ from $\mathcal{G}(H)$. Then

$$
\frac{|E(G)|}{|V(G)|} \leq \mu(H) \cdot \operatorname{VC}-\operatorname{dim}^{*}(G) \leq \mu(H) \cdot \log |V(G)| .
$$

We conjecture that in fact a stronger result holds:

Conjecture 1. Let $G$ be a subgraph of the Cartesian product $G_{1} \square \cdots \square G_{m}$. Then $\frac{|E(G)|}{|V(G)|} \leq$ VC-dens* $(G)$.

A partial evidence for this conjecture is Lemma 5 below showing that $\operatorname{VC}$-dens ${ }^{*}(G) \leq \frac{\mu(H)}{2}$. VC-dim* $(G)$.

As a direct consequence of [4, Lemma 3.1] and Theorem 3, we obtain the following: 
Corollary 1. If $G$ is a subgraph of a Cartesian product of connected graphs from $\mathcal{G}(H)$ and

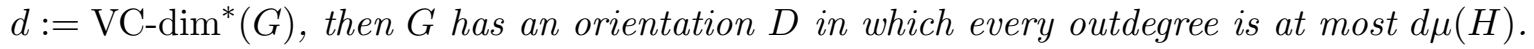

The proof of Theorem 2 is given in Section 5. The proof of Theorem 3 occupies Section 6 . where we also present basic properties of the VC-dimension and VC-density of subgraphs of Cartesian products.

\section{Proof of Theorem 2}

Let $n:=|V(G)|$. Since $\operatorname{dens}\left(G_{i}^{\prime}\right) \leq \operatorname{dens}\left(G_{i}\right)$ for any subgraph $G_{i}^{\prime}$ of $G_{i}$ for $i=1, \ldots, m$,

$$
\left\lceil\max \left\{\operatorname{dens}\left(\pi_{1}(G)\right), \ldots, \operatorname{dens}\left(\pi_{m}(G)\right)\right\}\right\rceil \leq\left\lceil\max \left\{\operatorname{dens}\left(G_{1}\right), \ldots, \operatorname{dens}\left(G_{m}\right)\right\}\right\rceil
$$

holds, establishing the second inequality $\beta_{0} \log |V(G)| \leq \beta \log |V(G)|$ (recall that $\pi_{i}(G)$ is the projection of $G$ on factor $G_{i}$ ). We prove the inequality

$$
\frac{|E(G)|}{|V(G)|} \leq \beta_{0} \log |V(G)|=\left\lceil\max \left\{\operatorname{mad}\left(\pi_{1}(G)\right), \ldots, \operatorname{mad}\left(\pi_{m}(G)\right)\right\}\right\rceil \cdot \log n
$$

by induction on $n$. If $n=1$, then we are obviously done. So suppose that $n \geq 2$. Then the projection $\pi_{i}(G)$ on some factor $G_{i}$ contains at least two vertices. From Lemma 1 , it follows that $\pi_{i}(G)$ has two vertices $v_{i}^{\prime}$ and $v_{i}^{\prime \prime}$ of degree at $\operatorname{most}\left\lceil\operatorname{mad}\left(\pi_{i}(G)\right)\right\rceil \leq \beta_{0}$. Denote by $A^{\prime}\left(\operatorname{resp} . A^{\prime \prime}\right)$ the set of all vertices of the graph $G$ having $v_{i}^{\prime}$ (resp. $v_{i}^{\prime \prime}$ ) as their $i$ th coordinate (see Fig 2 (b)). Since $v_{i}^{\prime}, v_{i}^{\prime \prime} \in V\left(\pi_{i}(G)\right)$, both $A^{\prime}$ and $A^{\prime \prime}$ are nonempty. At least one of the sets $A^{\prime}, A^{\prime \prime}$ contains at most $n / 2$ vertices, say $\left|A^{\prime}\right| \leq \frac{n}{2}$. Set $B:=V(G) \backslash A^{\prime}$ and denote by $G^{\prime}$ and $G^{\prime \prime}$ the subgraphs of $G$ induced by the sets $A^{\prime}$ and $B$. Let $\beta_{0}^{\prime}:=\left\lceil\max \left\{\operatorname{mad}\left(\pi_{1}\left(G^{\prime}\right)\right), \ldots, \operatorname{mad}\left(\pi_{m}\left(G^{\prime}\right)\right)\right\}\right\rceil$ and $\beta_{0}^{\prime \prime}:=\left\lceil\max \left\{\operatorname{mad}\left(\pi_{1}\left(G^{\prime \prime}\right)\right), \ldots, \operatorname{mad}\left(\pi_{m}\left(G^{\prime \prime}\right)\right)\right\}\right\rceil$. Since $\pi_{j}\left(G^{\prime}\right)$ and $\pi_{j}\left(G^{\prime \prime}\right)$ are subgraphs of $\pi_{i}(G)$, $j=1, \ldots, m, \beta_{0}^{\prime}, \beta_{0}^{\prime \prime} \leq \beta_{0}$. By induction assumption, $\left|E\left(G^{\prime}\right)\right| \leq \beta_{0}^{\prime}\left|A^{\prime}\right| \log \left|A^{\prime}\right| \leq \beta_{0}\left|A^{\prime}\right| \log \left|A^{\prime}\right|$ and $\left|E\left(G^{\prime \prime}\right)\right| \leq \beta_{0}^{\prime \prime}|B| \log |B| \leq \beta_{0}|B| \log |B|$. Since $A^{\prime}$ and $B$ partition $V(G), E(G)$ is the disjoint union of $E\left(G^{\prime}\right), E\left(G^{\prime \prime}\right)$, and $E\left(A^{\prime}, B\right)$, where $E\left(A^{\prime}, B\right)$ is the set of edges of $G$ with one end in $A^{\prime}$ and another end in $B$. Since any vertex of $A^{\prime}$ has at most $d\left(v_{i}^{\prime}\right) \leq\left\lceil\operatorname{mad}\left(\pi_{i}(G)\right)\right\rceil \leq \beta_{0}$ incident edges in $E\left(A^{\prime}, B\right)$, we obtain $\left|E\left(A^{\prime}, B\right)\right| \leq \beta_{0} \cdot\left|A^{\prime}\right|=\beta_{0}\left|A^{\prime}\right| \log 2$. Consequently,

$$
\begin{aligned}
|E(G)| & =\left|E\left(G^{\prime}\right)\right|+\left|E\left(G^{\prime \prime}\right)\right|+\left|E\left(A^{\prime}, B\right)\right| \\
& \leq \beta_{0}\left|A^{\prime}\right| \log \left|A^{\prime}\right|+\beta_{0}|B| \log |B|+\beta_{0}\left|A^{\prime}\right| \log 2 \\
& =\beta_{0}\left|A^{\prime}\right| \log \left(2\left|A^{\prime}\right|\right)+\beta_{0}|B| \log |B| \\
& \leq \beta_{0}\left(\left|A^{\prime}\right|+|B|\right) \log n=\beta_{0} n \log n .
\end{aligned}
$$

\section{Proof of Theorem 3}

The proof of Theorem 3 goes along the lines of the proof of Theorem 1 of [27] but is technically more involved. The roadmap of the proof is as follows. The first three results (Lemmas 4, 5, and 6) present the elementary properties of VC-dimension and VC-density and some relationships between them. To prove an analog of Lemma 3 we have to extend to subgraphs of products of graphs the operators $\mathcal{S}_{e}$ and $\mathcal{S}^{e}$ defined for set systems $\mathcal{S}$. For this, we pick two adjacent vertices $u$ and $v$ of some factor $G_{i}$ and define the graphs $G_{u v}$ and $G_{c}^{u v}$. The graph $G_{u v}$ is obtained from $G$ by contracting every edge of type $u v . G_{u v}$ is a subgraph of the Cartesian product having the same factors as $\Gamma=G_{1} \square \cdots \square G_{m}$, only the $i$ th factor is $G_{i}$ in which the edge $u v$ is contracted. The definition of the graph $G_{c}^{u v}$ is more involved and is given below. Then we prove the analogues of the inequalities $\mathrm{VC}-\operatorname{dim}\left(\mathcal{S}_{e}\right) \leq d$ and $\mathrm{VC}-\operatorname{dim}\left(\mathcal{S}^{e}\right) \leq d-1$ for graphs $G_{u v}$ and $G_{c}^{u v}(\operatorname{Lemmas} 7$ and 8). Finally, we have to obtain analogues of the equality $|\mathcal{S}|=\left|\mathcal{S}_{e}\right|+\left|\mathcal{S}^{e}\right|$ and of the inequality $|E(G)| \leq\left|E\left(G_{e}\right)\right|+\left|E\left(G^{e}\right)\right|+\left|V\left(G^{e}\right)\right|$ to the graphs $G, G_{u v}$, and $G_{c}^{u v}$. This is done in Lemma 9 . 
To proceed by induction and to prove that $\frac{|E(G)|}{|V(G)|} \leq \mu(H) \cdot \operatorname{VC}-\operatorname{dim}^{*}(G)$, in Lemma 10 we show that $\frac{\left|V\left(G^{u v}\right)\right|}{\left|V\left(G_{c}^{u v}\right)\right|} \leq|N| \leq \mu(H)-1$, where $N$ is the set of the common neighbors of $u$ and $v$. This is the case if one of the vertices $u$ or $v$ has degree $\leq \mu(H)$ (in our case, such a vertex always exists since each factor $G_{i}$ is $\mu(H)$-degenerated).

6.1. Properties of VC-dimension and VC-density. We continue with some basic properties of minor and induced VC-dimensions for products of arbitrary connected graphs and extend Lemma 3. In all these results, we suppose that $G$ is a subgraph of the Cartesian product $\Gamma:=\prod_{i=1}^{m} G_{i}=G_{1} \square \cdots \square G_{m}$ of connected graphs $G_{1}, \ldots, G_{m}$ and that $G$ has $n$ vertices. Since shattering in the definition of $\mathrm{VC}-\operatorname{dim}(G)$ and $\mathrm{VC}-\operatorname{dens}(G)$ is respect to subproducts and since all subproducts are minor-subproducts, we immediately obtain:

Lemma 4. VC-dim $(G) \leq \mathrm{VC}-\operatorname{dim}^{*}(G)$ and VC-dens $(G) \leq \mathrm{VC}-\operatorname{dens}{ }^{*}(G)$.

The following lemma justifies in part the formulation of Conjecture 1:

Lemma 5. VC-dens* $(G) \leq \frac{\mu(H)}{2} \cdot \operatorname{VC}-\operatorname{dim}^{*}(G)$.

Proof. Let $M=M_{i_{1}} \square \cdots \square M_{i_{k}}$ be a minor-subproduct of $\Gamma$ such that $\frac{|E(M)|}{|V(M)|}=$ VC-dens* $(G)$, and let $M^{\prime}=M_{j_{1}}^{\prime} \square \cdots \square M_{j_{h}}^{\prime}$ be a minor-subproduct such that $h=$ VC-dim* $(G)$. First, notice two things: (1) $h \geq k$ because, by definition, we chose $M$ with no trivial factors (so if $k$ was greater than $h$ we would have taken, at least, $\left.M^{\prime}=M\right) ;(2)$ a simple counting of the vertices and edges of a Cartesian product $\Gamma^{\prime}=G_{1}^{\prime} \square \cdots \square G_{m}^{\prime}$ shows that $\frac{\left|E\left(\Gamma^{\prime}\right)\right|}{\left|V\left(\Gamma^{\prime}\right)\right|}=\frac{\sum_{i=1}^{m}\left|E\left(G_{i}^{\prime}\right)\right| \cdot \prod_{j \neq i}\left|V\left(G_{j}^{\prime}\right)\right|}{\prod_{i=1}^{m}\left|V\left(G_{i}^{\prime}\right)\right|}=$ $\sum_{i=1}^{m} \frac{\left|E\left(G_{i}^{\prime}\right)\right|}{\left|V\left(G_{i}^{\prime}\right)\right|}$. We then have $\frac{|E(M)|}{|V(M)|}=\sum_{l=1}^{k} \frac{\left|E\left(M_{i_{l}}\right)\right|}{\left|V\left(M_{i_{l}}\right)\right|}$. Since we defined $\Gamma$ is a product of $H$-minor free graphs, for all $l \in\{1, \ldots, k\}, \frac{\left|E\left(M_{i_{l}}\right)\right|}{\left|V\left(M_{i_{l}}\right)\right|}=\frac{1}{2} d\left(M_{i_{l}}\right) \leq \frac{\mu(H)}{2}$. Then, $\frac{|E(M)|}{|V(M)|}=$ VC-dens $^{*}(G) \leq$ $\frac{\mu(H)}{2} \cdot k \leq \frac{\mu(H)}{2} \cdot h=\frac{\mu(H)}{2} \cdot \operatorname{VC}-\operatorname{dim}^{*}(G)$.

Lemma 6. $\operatorname{VC}-\operatorname{dim}^{*}(G) \leq \log n$.

Proof. Let $M=M_{1} \square \cdots \square M_{m}$ be a minor subproduct of $\prod_{i=1}^{m} G_{i}$ shattered by $G$ with $k=$ VC-dim* $(G)$ non-trivial factors. We want to show that $k \leq \log n$. Let $\mathcal{P}_{i}=\left\{P_{1}^{i}, \ldots, P_{t_{i}}^{i}\right\}$ denote the partition of $G_{i}$ defining the minor $M_{i}$. Since every non-trivial factor of $M$ contains at least two vertices, $\mathcal{P}:=\mathcal{P}_{1} \times \cdots \times \mathcal{P}_{m}$ contains at least $2^{k}$ parts. By the definition of shattering, any of those parts $P_{l_{1}}^{1} \times \cdots \times P_{l_{m}}^{m}$ (where $l_{1}=1, \ldots, t_{1}, \ldots$, and $\left.l_{m}=1, \ldots, t_{m}\right)$ contains a vertex $x$ of $G$. Since $\mathcal{P}$ is a partition of $\Gamma$, two vertices belonging to different parts have to be different. Consequently, $G$ contains at least $2^{k}$ vertices.

We continue with the extension to subgraphs of Cartesian products of the operators $\mathcal{S}_{e}$ and $\mathcal{S}^{e}$ defined for set systems $\mathcal{S}$. In case of set systems $\mathcal{S}$, the 1-inclusion graph $G(\mathcal{S})$ is an induced subgraph of the product of $K_{2}$ 's. Then $e$ corresponds to a factor of this product and $G\left(\mathcal{S}_{e}\right)$ can be viewed as the image of $G$ in the product of $K_{2}$ 's where the whole factor corresponding to $e$ was contracted. In case when the factors are arbitrary graphs, contracting a whole factor of the product would be too rough. So, let $u$ and $v$ be two adjacent vertices of some factor $G_{i}$. Let $N$ denote the set of common neighbors of $u$ and $v$ in $G_{i}$. Let $\widehat{G}_{i}$ be the graph obtained from $G_{i}$ by contracting the edge $u v$, namely, the graph in which the edge $u v$ is replaced by a vertex $w$ and every edge $x u$ and/or $x v$ of $G_{i}$ is replaced by a single new edge $x w$; thus $\widehat{G}_{i}$ does not contain loops and multiple edges. Let $\widetilde{G}_{i}$ be the graph which is a star having as the central vertex a vertex $\widetilde{w}$ corresponding to the edge $u v$ and as the set $\widetilde{N}$ of leaves the vertices $\widetilde{x}$ corresponding to vertices $x$ of $N$ (i.e., such that $x u v$ is a triangle of $G_{i}$ ); the edges of $\widetilde{G_{i}}$ are all pairs of the form $\widetilde{w} \widetilde{x}$. 


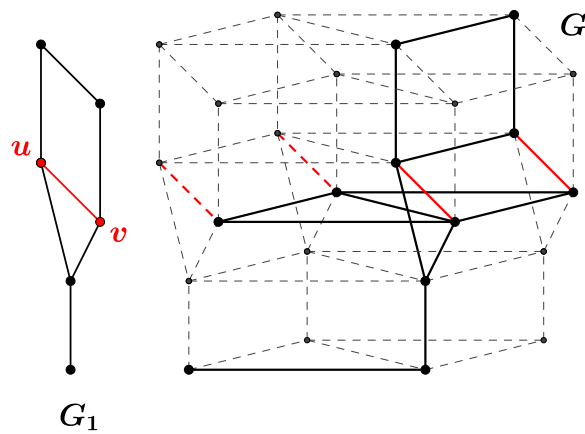

$G_{2}$

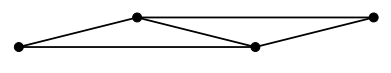

$G^{u v}$
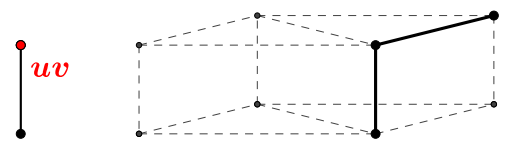

$\widetilde{G}_{1}$

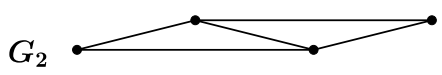

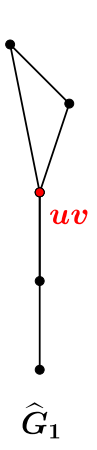

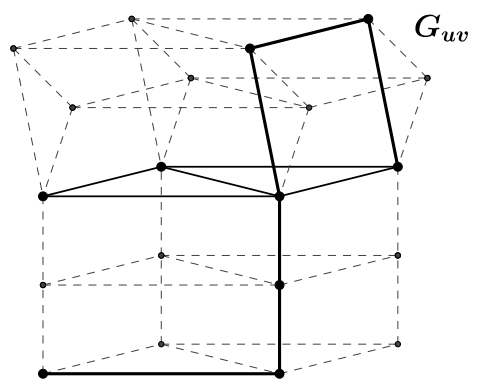

$\widehat{G}_{1}$

$G_{2}$

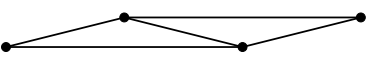

$G_{c}^{u v}$

$u v$

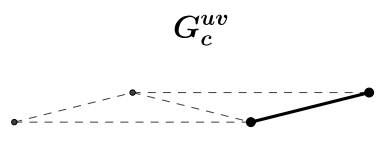

$\widetilde{G}_{1}^{\prime}$

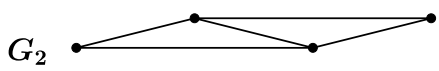

Figure 8. Examples of graphs $G_{u v}$ and $G_{c}^{u v}$

Let $G_{u v}$ be the subgraph of $\widehat{\Gamma}:=G_{1} \square \cdots \square G_{i-1} \square \widehat{G}_{i} \square G_{i+1} \square \cdots \square G_{m}$ obtained from $G$ by contracting every edge of type $u v$, i.e., by identifying any pair of vertices of the form $\left(\left(v_{1}, \ldots, v_{i-1}, u, v_{i+1}, \ldots, v_{m}\right),\left(v_{1}, \ldots, v_{i-1}, v, v_{i+1}, \ldots, v_{m}\right)\right)$, and removing multiple edges. Let $G^{u v}$ be the subgraph of $\widetilde{\Gamma}:=G_{1} \square \cdots \square G_{i-1} \square \widetilde{G}_{i} \square G_{i+1} \square \cdots \square G_{m}$ obtained from $G$ by applying the transformation of $G_{i}$ to $\widetilde{G}_{i}$. Namely, $G^{u v}$ is the subgraph of $\widetilde{\Gamma}$ induced by the following set of vertices: (1) $\left(v_{1}, \ldots, v_{i-1}, \widetilde{w}, v_{i+1}, \ldots, v_{m}\right)$ is a vertex of $G^{u v}$ if $\left(v_{1}, \ldots, v_{i-1}, u, v_{i+1}, \ldots, v_{m}\right)$ and $\left(v_{1}, \ldots, v_{i-1}, v, v_{i+1}, \ldots, v_{m}\right)$ are vertices of $G$ and $(2)\left(v_{1}, \ldots, v_{i-1}, \widetilde{x}, v_{i+1}, \ldots, v_{m}\right)$ is a vertex of $G^{u v}$ if $\left(v_{1}, \ldots, v_{i-1}, x, v_{i+1}, \ldots, v_{m}\right)$ is a vertex of $G, x \in N$, and $\left(v_{1}, \ldots, u, \ldots, v_{m}\right)$ and $\left(v_{1}, \ldots, v, \ldots, v_{m}\right)$ are vertices of $G$.

Notice that $G_{u v}$ plays the role of $\mathcal{S}_{e}$ in the binary case. To play the role of $\mathcal{S}^{e}$ we define the graph $G_{c}^{u v}$ which is the subgraph of $G^{u v}$ induced by the vertices that have a central node $\widetilde{w}$ of $\widetilde{G}_{i}$ as their $i$ th coordinate. If $G_{i}$ is a $K_{2}$ (or, more generally, the edge $u v$ does not belong to a triangle), then $G^{u v}$ coincides with $G_{c}^{u v}$. The remaining vertices of $G^{u v}$, those having a leaf $\widetilde{x}$ of $\widetilde{G}_{i}$ as their $i$ th coordinate, will be called tip vertices. We denote by $V_{l}\left(G^{u v}\right)$ the set of tip vertices. Finally, the remaining edges of $G^{u v}$ will be denoted by $E_{l}\left(G^{u v}\right)$. Examples of graphs $G_{u v}$ and $G_{c}^{u v}$ are presented in Fig. 8. In all subsequent results, we suppose that $G$ is a subgraph of a Cartesian product $\Gamma:=G_{1} \square \cdots \square G_{m}, G_{i}$ is any factor of $\Gamma$, and $u v$ is any edge of $G_{i}$.

Lemma 7. Let $\Gamma=G_{1} \square \cdots \square G_{m}$ be a Cartesian product of finite graphs and let $G$ be an induced subgraph of $\Gamma$. For all $G_{i}, i \in\{1, \ldots, m\}$, and for all $u v \in E\left(G_{i}\right)$, the following inequalities hold:

(1) $\mathrm{VC}-\operatorname{dim}^{*}\left(G_{u v}\right) \leq \mathrm{VC}-\operatorname{dim}^{*}(G)$;

(2) VC-dens* $\left(G_{u v}\right) \leq \mathrm{VC}-\operatorname{dens}^{*}(G)$,

where the $V C$-dimension and the $V C$-density of $G_{u v}$ are computed with respect to $\widehat{\Gamma}$. 
Proof. We will prove that if a minor-subproduct $M$ of $\widehat{\Gamma}$ is shattered by $G_{u v}$, then $M$ is also a minor-subproduct of $\Gamma$ shattered by $G$. Let $M:=M_{1} \square \cdots \square M_{m}$ be defined by the partition $\mathcal{P}:=\mathcal{P}_{1} \times \cdots \times \mathcal{P}_{m}$ of the product $\widehat{\Gamma}$, where $\mathcal{P}_{j}:=\left\{P_{1}^{j}, \ldots, P_{t_{j}}^{j}\right\}$ is a partition of $G_{j}$ (or $\widehat{G}_{i}$ if $j=i$ ) defining the minor $M_{j}, j=1, \ldots, m$. We suppose that $M$ contains $k$ non-trivial factors indexed by $i_{1}, \ldots, i_{k}$. Since $M$ is shattered by $G_{u v}$, any set $P_{l_{1}}^{1} \times \cdots \times P_{l_{m}}^{m}$ of $\mathcal{P}$ (where $\left.l_{1}=1, \ldots, t_{1}, \ldots, l_{m}=1, \ldots, t_{m}\right)$ contains a vertex $x$ of $G_{u v}$. Recall that we merged two adjacent vertices $u$ and $v$ of the factor $G_{i}$ of $\Gamma$. We distinguish two cases.

Case 1. $i \notin\left\{i_{1}, \ldots, i_{k}\right\}$.

Then $M$ is also a minor-subproduct of $\Gamma$. We will prove that the vertex $x$ of $P_{l_{1}}^{1} \times \cdots \times P_{l_{m}}^{m}$ also belongs to $G$. Consider the $i$ th coordinate $x_{i}$ of $x$. If $x_{i} \neq w$, then $x$ also belongs to $G$. Otherwise, if $x_{i}=w$, then at least one of the vertices $\left(x_{1}, \ldots, u, \ldots, x_{m}\right)$ or $\left(x_{1}, \ldots, v, \ldots, x_{m}\right)$ must be a vertex of $G$, and thus we can denote by $x$ that vertex.

Case 2. $i \in\left\{i_{1}, \ldots, i_{k}\right\}$, say $i:=i_{j}$.

Consider the partition $\mathcal{P}_{i}:=\left\{P_{1}^{i}, \ldots, P_{t_{i}}^{i}\right\}$ of $\widehat{G}_{i}=\widehat{G}_{i_{j}}$ and suppose that $w \in P_{l}^{i}$. Using the partition $\mathcal{P}_{i}$ of $\widehat{G}_{i}$, we will define in the following way the partition $\mathcal{P}_{i}^{\prime}=\left\{P_{1}^{i}, \ldots, P_{l-1}^{i}, P^{\prime}, \ldots, P_{t_{i}}^{i}\right\}$ of $G_{i}$, where $P^{\prime}:=P_{l}^{i} \backslash\{w\} \cup\{u, v\}$. Let $\mathcal{P}^{\prime}=\mathcal{P}_{1} \times \cdots \times \mathcal{P}_{i-1} \times \mathcal{P}_{i}^{\prime} \times \mathcal{P}_{i+1} \times \cdots \times \mathcal{P}_{m}$. It can be easily seen that $\mathcal{P}^{\prime}$ is a partition of the Cartesian product $\Gamma$, and that it provides a minorsubproduct representation of $M$ (in the product $\Gamma$ ). Then as in Case 1 one can show that either $x_{i} \neq w$ and $x$ is also a vertex of $\Gamma$ belonging to $G$, or that $x_{i}=w$ and $\left(x_{1}, \ldots, u, \ldots, x_{m}\right)$, or $\left(x_{1}, \ldots, v, \ldots, x_{m}\right)$ must belong to $G$.

This shows that if $M$ is a minor-subproduct of $\widehat{\Gamma}$ shattered by $\widehat{G}$, then $M$ is also a minorsubproduct of $\Gamma$ shattered by $G$, establishing the inequalities (1) and (2).

Lemma 8. Let $\Gamma=G_{1} \square \cdots \square G_{m}$ be a Cartesian product of finite graphs and let $G$ be an induced subgraph of $\Gamma$. For all $G_{i}, i \in\{1, \ldots, m\}$, and for all $u v \in E\left(G_{i}\right)$, the following inequalities hold:

(1) $\mathrm{VC}-\operatorname{dim}^{*}\left(G_{c}^{u v}\right) \leq \mathrm{VC}-\operatorname{dim}^{*}(G)-1$;

(2) $\mathrm{VC}_{\text {-dens }}^{*}\left(G_{c}^{u v}\right) \leq \mathrm{VC}-\operatorname{dens}^{*}(G)-1$,

where the $V C$-dimension and the $V C$-density of $G_{c}^{u v}$ are computed with respect to $\widetilde{\Gamma}$.

Proof. Suppose by way of contradiction that $G_{c}^{u v}$ shatters in $\widetilde{\Gamma}$ a minor-product $M=$ $M_{1} \square \cdots \square M_{m}$ such that either $\frac{|E(M)|}{|V(M)|} \geq$ VC-dens* $^{*}(G)$ or $M$ has at least VC-dim* $(G)$ nontrivial factors $M_{i_{1}}, \ldots, M_{i_{k}}$. Let $M$ be defined by the partition $\mathcal{P}:=\mathcal{P}_{1} \times \cdots \times \mathcal{P}_{m}$ of $\widetilde{\Gamma}$, where $\mathcal{P}_{j}:=\left\{P_{1}^{j}, \ldots, P_{t_{j}}^{j}\right\}$ is a partition of $G_{j}$ (or $\widetilde{G}_{i}$ if $j=i$ ) defining the minor $M_{j}, j=1, \ldots, m$.

Notice that all vertices of $G_{c}^{u v}$ have $\widetilde{w}$ as their $i$ th coordinate. Therefore if $i \notin\left\{i_{1}, \ldots, i_{k}\right\}$, we can define $\mathcal{P}^{\prime}$ as the product of partitions $\mathcal{P}$ where the $i$ th partition $\mathcal{P}_{i}=V\left(\widetilde{G}_{i}\right)$ has been replaced by $\mathcal{P}_{i}^{\prime}=V\left(G_{i}\right)$. So $\mathcal{P}^{\prime}$ is a partition of $\Gamma$ defining the same minor $M$ now shattered by $G$. Vice-versa, if $i \in\left\{i_{1}, \ldots, i_{k}\right\}$, say $i=i_{k}$, then, since all vertices of $G_{c}^{u v}$ have $\widetilde{w}$ as their $i$ th coordinate, the partition $\mathcal{P}_{i}$ must contain a single member, i.e., $M_{i}=K_{1}$. Thus further we can assume that $\mathcal{P}_{i}=V\left(\widetilde{G}_{i}\right)$, and $i \notin\left\{i_{1}, \ldots, i_{k}\right\}$.

Now, we assert that $M^{\prime}:=M_{1} \square \cdots \square M_{i-1} \square K_{2} \square M_{i+1} \square \cdots \square M_{m}$ is a minor-subproduct of $\Gamma$ shattered by the graph $G$. Since $M$ has $K_{1}$ as $i$ th coordinate, this would imply that $\left|V\left(M^{\prime}\right)\right|=$ $2|V(M)|$ and $\left|E\left(M^{\prime}\right)\right|=2|E(M)|+|V(M)|$, whence

$$
\frac{\left|E\left(M^{\prime}\right)\right|}{\left|V\left(M^{\prime}\right)\right|}=\frac{2|E(M)|+|V(M)|}{2|V(M)|}=\frac{|E(M)|}{|V(M)|}+\frac{1}{2}=\operatorname{VC}-d e n s^{*}\left(G_{c}^{u v}\right)+\frac{1}{2} \geq \operatorname{VC}^{\prime} \text { dens }{ }^{*}(G)+\frac{1}{2},
$$


leading to a contradiction with $\frac{|E(M)|}{|V(M)|} \geq \mathrm{VC}-\operatorname{dens}^{*}(G)$ and showing 22. Furthermore, $M^{\prime}=$ $M \square K_{2}$ implies that its VC-dim* $\left(M^{\prime}\right)=\mathrm{VC}-\operatorname{dim}^{*}(M)+1$ leading to a contradiction with $k \geq$ VC-dim* $(G)$ and showing (1).

First we prove that $M^{\prime}$ is a minor-product of $\Gamma$. Recall that $M=M_{1} \square \cdots \square M_{m}$ is defined by the partition $\mathcal{P}:=\mathcal{P}_{1} \times \cdots \times \mathcal{P}_{m}$ of $\widetilde{\Gamma}$, where $\mathcal{P}_{i}=V\left(\widetilde{G}_{i}\right)$. Define the following partition $\mathcal{P}^{\prime}:=\mathcal{P}_{1} \times \cdots \times \mathcal{P}_{i-1} \times \mathcal{P}_{i}^{\prime} \times \mathcal{P}_{i+1} \times \cdots \times \mathcal{P}_{m}$ of $\Gamma$, where $\mathcal{P}_{i}^{\prime}=\left\{P_{1}^{i}, P_{2}^{i}\right\}$ and $\left\{P_{1}^{i}, P_{2}^{i}\right\}$ define a partition of $V\left(G_{i}\right)$ into two connected subgraphs, the first containing the vertex $u$ and the second containing the vertex $v$. This can be done by letting $P_{1}^{i}$ be the set of all vertices of $G_{i}$ reachable from $u$ via simple paths not passing via $v$ and by setting $P_{2}^{i}:=V\left(G_{i}\right) \backslash P_{1}^{i}$. Then clearly $M^{\prime}$ is defined by the partition $\mathcal{P}^{\prime}$.

It remains to show that $M^{\prime}$ is shattered in $\Gamma$ by $G$. Pick any set $P_{l_{1}}^{1} \times \cdots \times P_{l_{m}}^{m}$ of $\mathcal{P}^{\prime}$ (where $l_{1}=1, \ldots, t_{1}, \ldots, l_{m}=1, \ldots, t_{m}$ and $t_{i}=2$ ). Since $M$ is shattered by $G_{c}^{u v}$, the set $P_{l_{1}}^{1} \times \cdots \times P_{l_{i-1}}^{i-1} \times V\left(\widetilde{G}_{i}\right) \times P_{l_{i+1}}^{i+1} \times \cdots \times P_{l_{m}}^{m}$ has a vertex $x$ belonging to $G_{c}^{u v}$, and then having $\widetilde{w}$ as $i$ th coordinate. From the definition of $G_{c}^{u v}$ and of the vertex $\widetilde{w}$ we conclude that the vertex $x_{1}$ obtained from $x$ by replacing the $i$ th coordinate $\widetilde{w}$ by $u$ is a vertex of $P_{l_{1}}^{1} \times \cdots \times P_{1}^{i} \times \cdots \times P_{l_{m}}^{m}$, while the vertex $x_{2}$ obtained from $x$ by replacing the $i$ th coordinate $\widetilde{w}$ by $v$ is a vertex of $P_{l_{1}}^{1} \times \cdots \times P_{2}^{i} \times \cdots \times P_{l_{m}}^{m}$. Since $x$ is a vertex of $G_{c}^{u v}$, then $x_{1}$ and $x_{2}$ are vertices of $G$. This shows that both sets $P_{l_{1}}^{1} \times \cdots \times P_{1}^{i} \times \cdots \times P_{l_{m}}^{m}$ and $P_{l_{1}}^{1} \times \cdots \times P_{2}^{i} \times \cdots \times P_{l_{m}}^{m}$ of $\mathcal{P}^{\prime}$ contain vertices $x_{1}$ and $x_{2}$ which belong to $G$. This proves that $G$ shatters $M^{\prime}$ in $\Gamma$.

Lemma 9. The graphs $G, G_{u v}, G_{c}^{u v}$, and $G^{u v}$ satisfy the following relations:

$$
\left\{\begin{array}{l}
|V(G)|=\left|V\left(G_{u v}\right)\right|+\left|V\left(G^{u v}\right)\right|-\left|V_{l}\left(G^{u v}\right)\right|=\left|V\left(G_{u v}\right)\right|+\left|V\left(G_{c}^{u v}\right)\right|, \\
|E(G)| \leq\left|E\left(G_{u v}\right)\right|+\left|E\left(G^{u v}\right)\right|+\left|V\left(G_{c}^{u v}\right)\right| .
\end{array}\right.
$$

Proof. $G_{u v}$ contains the vertices of $G$ minus one vertex for each contracted edge of type $u v . G^{u v}$ contains one vertex for each contracted edge of $G$ (the vertices of $V\left(G_{c}^{u v}\right)$ ) plus one vertex for each triangle of $G$ involving an edge of type $u v$ (the vertices of $V_{l}\left(G^{u v}\right)$ ). Therefore to obtain $|V(G)|$, from $\left|V\left(G_{u v}\right)\right|+\left|V\left(G^{u v}\right)\right|$ we have to subtract $\left|V_{l}\left(G^{u v}\right)\right|$.

$E\left(G_{u v}\right)$ contains the set $E(G)$ of edges of $G$ minus (1) the contracted edges of type $u v$, minus (2) the multiple edges obtained when contracting a triangle of $G$ containing an edge of type $u v$, minus (3) the multiple edges obtained when contracting a square of $G$ with two opposite edges of type $u v$, and plus (4) some edges we may have created if we had only two opposite vertices of a square, one of type $u$ and the other of type $v$. Notice that there are $\left|V\left(G_{c}^{u v}\right)\right|$ edges of type $u v$ (group (1)), there are $\left|E_{l}\left(G^{u v}\right)\right|$ in group (2), and $\left|E\left(G_{c}^{u v}\right)\right|$ in group (3). Since $\left|E\left(G^{u v}\right)\right|=\left|E_{l}\left(G^{u v}\right)\right|+\left|E\left(G_{c}^{u v}\right)\right|$, we obtain the required inequality $|E(G)| \leq\left|E\left(G_{u v}\right)\right|+$ $\left|E\left(G^{u v}\right)\right|+\left|V\left(G_{c}^{u v}\right)\right|$.

Lemma 10. $\frac{\left|V\left(G^{u v}\right)\right|}{\left|V\left(G_{c}^{u v}\right)\right|} \leq|N|$, where $N$ is the set of the common neighbors of $u$ and $v$.

Proof. Each vertex of $G^{u v}$ is either a central vertex of the form $\left(v_{1}, \ldots, v_{i-1}, \widetilde{w}, v_{i+1}, \ldots, v_{m}\right)$ or is a tip vertex of the form $\left(v_{1}, \ldots, v_{i-1}, \widetilde{x}, v_{i+1}, \ldots, v_{m}\right)$. Each tip vertex of $G^{u v}$ is adjacent to a single central vertex. Since each central vertex of $G^{u v}$ is adjacent to at most $|N|$ tip vertices, we obtain the required inequality.

6.2. Proof of Theorem 3. Let $G$ be a subgraph with $n$ vertices of the Cartesian product $\Gamma:=G_{1} \square \cdots \square G_{m}$ of connected graphs $G_{1}, \ldots, G_{m}$ from $\mathcal{G}(H)$. We have to prove that $\frac{|E(G)|}{|V(G)|} \leq$ $\mu(H) \cdot \operatorname{VC}-\operatorname{dim}^{*}(G) \leq \mu(H) \cdot \log n$. The second inequality follows from Lemma 6 . We will prove the inequality $\frac{|E(G)|}{|V(G)|} \leq \mu(H) \cdot \mathrm{VC}-\operatorname{dim}^{*}(G)$ by induction on the number of vertices in the factors of $\Gamma$. Since each factor $G_{i}$ of $\Gamma$ belongs to $\mathcal{G}(H), G_{i}$ is $\mu(H)$-degenerated, i.e., $G_{i}$ and any of its 
subgraphs contains a vertex $v$ of degree at most $\mu(H)$. Let $u$ be any neighbor of $v$ in $G_{i}$. Then the set $N$ of common neighbors of $u$ and $v$ has size at most $\mu(H)-1$. Consider the graphs $G_{u v}, G^{u v}$, and $G_{c}^{u v}$ obtained from $G$ by performing the operations from previous subsection with respect to the edge $u v$ of $G_{i}$. Then $G_{u v}$ is a subgraph of the product $\widehat{\Gamma}=G_{1} \square \cdots \square G_{i-1} \square \widehat{G}_{i} \square G_{i+1} \cdots \square G_{m}$. Since $\widehat{G}_{i}$ is a minor of $G_{i}$, all factors of $\widehat{\Gamma}$ belong to $\mathcal{G}(H)$. Moreover, since $\widehat{G}_{i}$ contains fewer vertices than $G_{i}$, we can apply the induction assumption to subgraphs of $\widehat{\Gamma}$, in particular to $G_{u v}$. Analogously, $G^{u v}$ and $G_{c}^{u v}$ are subgraphs of the product $\widetilde{\Gamma}=G_{1} \square \cdots \square G_{i-1} \square \widetilde{G}_{i} \square G_{i+1} \square \cdots \square G_{m}$ and since $\widetilde{G}_{i}$ is a star isomorphic to a subgraph of $G_{i}$, all factors of $\widetilde{\Gamma}$ also belong to $\mathcal{G}(H)$. Since $\widetilde{G}_{i}$ contains fewer vertices than $G_{i}$, also the graphs $G^{u v}$ and $G_{c}^{u v}$ do. Consequently, we have $\frac{\left|E\left(G_{u v}\right)\right|}{\left|V\left(G_{u v}\right)\right|} \leq \mu(H) \cdot \operatorname{VC}-\operatorname{dim}^{*}\left(G_{u v}\right)$ and $\frac{\left|E\left(G_{c}^{u v}\right)\right|}{\left|V\left(G_{c}^{u v}\right)\right|} \leq \mu(H) \cdot \operatorname{VC}-\operatorname{dim}^{*}\left(G_{c}^{u v}\right)$.

By Lemma 9 and using the inequality $\frac{a_{1}+a_{2}}{b_{1}+b_{2}} \leq \max \left\{\frac{a_{1}}{b_{1}}, \frac{a_{2}}{b_{2}}\right\}$, we obtain

$$
\begin{aligned}
\frac{|E(G)|}{|V(G)|} & \leq \frac{\left|E\left(G_{u v}\right)\right|+\left|E\left(G^{u v}\right)\right|+\left|V\left(G_{c}^{u v}\right)\right|}{\left|V\left(G_{u v}\right)\right|+\left|V\left(G_{c}^{u v}\right)\right|} \\
& \leq \max \left\{\frac{\left|E\left(G_{u v}\right)\right|}{\left|V\left(G_{u v}\right)\right|}, \frac{\left|E\left(G^{u v}\right)\right|+\left|V\left(G_{c}^{u v}\right)\right|}{\left|V\left(G_{c}^{u v}\right)\right|}\right\} .
\end{aligned}
$$

By Lemma 7, VC-dim* $\left(G_{u v}\right) \leq \mathrm{VC}-\operatorname{dim}^{*}(G)$, whence

$$
\frac{\left|E\left(G_{u v}\right)\right|}{\left|V\left(G_{u v}\right)\right|} \leq \mu(H) \cdot \operatorname{VC}-\operatorname{dim}^{*}\left(G_{u v}\right) \leq \mu(H) \cdot \operatorname{VC}-\operatorname{dim}^{*}(G) \text {. }
$$

Thus it remains to provide a similar upper bound for $\frac{\left|E\left(G^{u v}\right)\right|+\left|V\left(G_{c}^{u v}\right)\right|}{\left|V\left(G_{c}^{u v}\right)\right|}$. Since $\left|E\left(G^{u v}\right)\right|+$ $\left|V\left(G_{c}^{u v}\right)\right|=\left|E\left(G_{c}^{u v}\right)\right|+\left|V_{l}\left(G^{u v}\right)\right|+\left|V\left(G_{c}^{u v}\right)\right|=\left|E\left(G_{c}^{u v}\right)\right|+\left|V\left(G^{u v}\right)\right|$ and $|N| \leq \mu(H)$, from Lemmas 8 and 10 we conclude:

$$
\begin{aligned}
\frac{\left|E\left(G^{u v}\right)\right|+\left|V\left(G_{c}^{u v}\right)\right|}{\left|V\left(G_{c}^{u v}\right)\right|} & =\frac{\left|E\left(G_{c}^{u v}\right)\right|+\left|V\left(G^{u v}\right)\right|}{\left|V\left(G_{c}^{u v}\right)\right|} \\
& \leq \mu(H) \cdot \operatorname{VC}_{-} \operatorname{dim}^{*}\left(G_{c}^{u v}\right)+\mu(H) \\
& \leq \mu(H) \cdot\left(\mathrm{VC}-d_{i m}^{*}(G)-1\right)+\mu(H) \\
& =\mu(H) \cdot \operatorname{VC}-\operatorname{dim}^{*}(G) .
\end{aligned}
$$

This establishes the inequality $\frac{|E(G)|}{|V(G)|} \leq \mu(H) \cdot \mathrm{VC}-\operatorname{dim}^{*}(G)$ and concludes the proof of the theorem.

Remark 4. To prove Conjecture 1, in Lemma 8 it is necessary to establish a stronger inequality (2).

\section{SPECIAL GRAPH ClASSES}

In this section we specify our results (and sometimes obtain stronger formulations) for subgraphs of Cartesian products of several classes of graphs: bounded degeneracy graphs, graphs with polylogarithmic average degree, dismantlable graphs, chordal graphs, octahedra, cliques.

7.1. Bounded degeneracy graphs. The degeneracy of a graph $G$ is the smallest $k$ such that the vertices of $G$ admit a total order $v_{1}, \ldots, v_{n}$ such that the degree of $v_{i}$ in the subgraph of $G$ induced by $v_{i}, \ldots, v_{n}$ is at most $k$ (similarly to arboricity, degeneracy of $G$ is linearly bounded by its density $\operatorname{dens}(G)$ ). Let $\mathcal{G}_{\delta}$ denote the class of all graphs with degeneracy at most $\delta$. $\mathcal{G}_{\delta}$ contains the class of all graphs in which all degrees of vertices are bounded by $\delta$. $\mathcal{G}_{5}$ contains the class of all planar graphs. The density of any graph from $\mathcal{G}_{\delta}$ is bounded by $\delta$. From Theorem 2 we immediately obtain the following corollary: 
Corollary 2. If $G$ is a subgraph of $G_{1} \square \cdots \square G_{m}$ and $G_{1}, \ldots, G_{m} \in \mathcal{G}_{\delta}$, then $\frac{|E(G)|}{|V(G)|} \leq 2 \delta$. $\log |V(G)|$.

7.2. Graphs of polylogarithmic average degree. Let $\mathcal{G}_{\log , k}$ denote the class of graphs $H$ closed by subgraphs and in which mad is bounded by a polylogarithmic function, i.e., $2\lceil\operatorname{dens}(H)\rceil \leq \log ^{k}|V(H)|$. In this case, Theorem 2 provides the following corollary:

Corollary 3. If $G$ is a subgraph of $G_{1} \square \cdots \square G_{m}$ and $G_{1}, \ldots, G_{m} \in \mathcal{G}_{\log , k}$, then $G \in \mathcal{G}_{\log , k+1}$, i.e., $\frac{|E(G)|}{|V(G)|} \leq \log ^{k+1}|V(G)|$.

Proof. By Theorem $2, \frac{|E(G)|}{|V(G)|} \leq \max \left\{\left\lceil\operatorname{mad}\left(\pi_{1}(G)\right)\right\rceil, \ldots,\left\lceil\operatorname{mad}\left(\pi_{m}(G)\right)\right\rceil\right\} \cdot \log |V(G)|$. Since $\pi_{i}(G)$ is a subgraph of $G_{i}$, all projections $\pi_{i}(G), i=1, \ldots, m$, of $G$ on factors belong to $\mathcal{G}_{\log , k}$. Consequently, $\left\lceil\operatorname{mad}\left(\pi_{i}(G)\right)\right\rceil \leq \log ^{k}\left|V\left(\pi_{i}(G)\right)\right| \leq \log ^{k}|V(G)|$. Thus $\frac{|E(G)|}{|V(G)|} \leq \log ^{k+1}|V(G)|$.

7.3. Products of dismantlable graphs. A vertex $u$ of a graph $G$ is dominated in $G$ by its neighbor $v$ if $N[u] \subseteq N[v]$ (uv is called a dominating edge). A graph $G$ is dismantlable [30 if $G$ admits an ordering $v_{1}, v_{2}, \ldots, v_{n}$ of its vertices such that for each $i \in\{1, \ldots, n\}, v_{i}$ is dominated in the subgraph $G\left[v_{i}, \ldots, v_{n}\right]$ of $G$ induced by the vertices $v_{i}, \ldots, v_{n}$. Dismantlable graphs comprise chordal graphs, bridged graphs, and weakly bridged graphs as subclasses (for definition of the last two classes and their dismantlability see for example 11]). Dismantlable graphs are exactly the cop-win graphs 38.

A min-dismantling order of $G$ is a dismantling order in which at each step $i, v_{i}$ is a dominated vertex of $G\left[v_{i}, \ldots, v_{n}\right]$ of smallest degree. We define the dismantling degeneracy $\operatorname{dd}(G)$ of $G$ as the maximum degree of a vertex $v_{i}$ in $G\left[v_{i}, \ldots, v_{n}\right]$ in a min-dismantling order of $G$. Let $\Gamma:=\prod_{i=1}^{m} G_{i}$ be a Cartesian product of dismantlable graphs $G_{1}, \ldots, G_{m}$. Let $v_{i, 1}, \ldots, v_{i, n_{i}}$ be a min-dismantling ordering $<_{i}$ of the vertex-set of $G_{i}, i=1, \ldots, m$. Define a partial order $\left(\prod_{i=1}^{m} V_{i}, \preceq\right)$ on the vertex set of $\Gamma$ as the Cartesian product of the totally ordered sets $\left(V_{i},<_{i}\right)$, $i=1, \ldots, m$. Let $<$ denote any linear extension of $\preceq$. We will call this order a product mindismantling order. The maximal degree of a vertex $v_{i}$ in the subgraph of $\Gamma$ induced by $v_{i}, \ldots, v_{N}$, where $N=n_{1} \times \cdots \times n_{m}$, will be denoted by $\operatorname{dd}^{*}(\Gamma)$. One can show that $\operatorname{dd}^{*}(\Gamma)=\sum_{i} \operatorname{dd}\left(G_{i}\right)$.

For a subgraph $G$ of $\Gamma$ with its vertices ordered $v_{1}, \ldots, v_{n}$ according to the total order $<$ on $\Gamma$, we will define $\operatorname{dd}^{*}(G)$ as the maximum degree of a vertex $v_{i}$ in the subgraph of $G$ induced by $v_{i}, \ldots, v_{n}$. Note that $\operatorname{dd}^{*}(G) \leq \operatorname{dd}^{*}(\Gamma)$. Note also that contracting a dominating edge $u_{i} v_{i}$ of a factor $G_{i}$ gives a minor of $G_{i}$ that, at the same time, is an induced subgraph of $G_{i}$. Thus, if $G$ is a subgraph of a Cartesian product of dismantlable graphs and at each step in the proof of Theorem 3 we contract a dominating edge of a factor, we obtain the following result:

Proposition 1. If $G$ is a subgraph of $\Gamma=G_{1} \square \cdots \square G_{m}$ and $G_{1}, \ldots, G_{m}$ are dismantlable graphs, then $\frac{|E(G)|}{|V(G)|} \leq \operatorname{dd}^{*}(G) \cdot \operatorname{VC}-\operatorname{dim}(G)$.

Proof. Since $G^{u v}$ is a subgraph of $G$, from Lemma 10 we infer that $\frac{\left|V\left(G^{u v}\right)\right|}{\left|V\left(G_{c}^{u v}\right)\right|} \leq \operatorname{dd}^{*}\left(G^{u v}\right) \leq \operatorname{dd}^{*}(G)$. Since $G_{u v}$ is an induced subgraph of $G$, we directly obtain that VC-dim $\left(G_{u v}\right) \leq \mathrm{VC}-\operatorname{dim}(G)$. Then, according to Lemma 8 for $\operatorname{VC}-\operatorname{dim}(G)$, we have $\operatorname{VC}-\operatorname{dim}\left(G_{c}^{u v}\right) \leq \mathrm{VC}-\operatorname{dim}(G)-1$. The result now follows from those remarks together with the previous inequality.

7.4. Products of chordal graphs. For chordal graphs $G$, we use the facts that any simplicial ordering of $G$ is a dominating ordering and that for any edge $u v$ of a chordal graph $G$, the graph $G_{u v}$ is chordal and $\omega\left(G_{u v}\right) \leq \omega(G)$ (where $\omega(G)$ is the size of a largest clique of $G$ ). As for dismantlable graphs, contracting each time an edge between a simplicial vertex and its neighbor and applying this property, we obtain the following corollary of Proposition 1 and Theorem 3 . 
Corollary 4. If $G$ is a subgraph of $G_{1} \square \cdots \square G_{m}$ and $G_{1}, \ldots, G_{m}$ are chordal graphs, then $\frac{|E(G)|}{|V(G)|} \leq \omega(G) \cdot$ VC-dim $(G)$.

One basic class of Cartesian products of chordal graphs is the class of Hamming graphs, i.e., Cartesian product of complete graphs. Consequently, Corollary 4 also applies to subgraphs of Hamming graphs. There is a lot of literature on this class of graphs, since it naturally generalize binary words of constant length (hypercubes) to words in arbitrary alphabets. In particular, generalizations of shattering and "VC-dimension" have been studied in the case of Hamming graphs (see [37, 39]), leading to a "stronger" Sauer's lemma [28] and density results [40].

7.5. Products of octahedra. The $d$-dimensional octahedron $K_{2, \ldots, 2}$ (or the cocktail party graph [17]) is the complete graph on $2 d$ vertices minus a perfect matching. Equivalently, $K_{2, \ldots, 2}$ is the $d$-partite graph in which each part has two vertices (which we will call opposite). $K_{2, \ldots, 2}$ is also the 1-skeleton of the $d$-dimensional cross polytope. If $e_{1}$ and $e_{2}$ are opposite vertices, it will be convenient to denote this by $e_{2}=\overline{e_{1}}$. A subgraph of an octahedron is called a suboctahedron. The d-dimensional octahedron $K_{2, \ldots, 2}$ can be viewed as the graph of an alphabet $\Sigma=\left\{e_{1}, \overline{e_{1}}, \ldots, e_{d}, \overline{e_{d}}\right\}$ with an involution $\varphi\left(e_{i}\right)=\overline{e_{i}}$ for each $i=1, \ldots, d$ (an involution on a finite set $X$ is a mapping $\varphi: X \rightarrow X$ such that $\varphi^{2}(x)=x$ for any $x \in X$ ). Consequently, the Cartesian product of $m d$-dimensional octahedra can be viewed as the graph of words of length $m$ of $(\Sigma, \varphi)$.

In this subsection, we show that if $G$ is a subgraph of a Cartesian product $\Gamma$ of $m$ octahedra $G_{1}, \ldots, G_{m}$ of respective dimensions $d_{1}, \ldots, d_{m}$, then an analog of Corollary 4 holds (for edgeisoperimetric problem in products of octahedra, see [25]). The difference to chordal graphs is that contracting an edge of an octahedron can increase its largest clique and the result is not a suboctahedron anymore. Therefore, we have to define the graphs $G_{u v}$ and $G^{u v}$ differently. If at the beginning all factors are octahedra, after a few steps they will no longer be octahedra but suboctahedra. If some factor is not a clique, then it contains two opposite vertices $e$ and $\bar{e}$ and we can identify them, transforming this factor into a suboctahedron with fewer opposite pairs. If all factors are cliques, then their product is a Hamming graph (which can be viewed as our induction basis) and we can use the results of previous subsection, namely Corollary 4 .

Let $G_{i}$ be a factor of the current Cartesian product $\Gamma$ and suppose that $G_{i}$ is a suboctahedron containing two opposite vertices $e$ and $\bar{e}$. Let $G$ be a subgraph of $\Gamma$. We will denote by $v^{i}$ a vertex of $\Gamma$ with all components fixed excepted the $i$ th one, i.e., $v^{i}$ fixes the position of a copy of $G_{i}$. We will denote this vertex by $v^{i}[e], v^{i}[\bar{e}]$, or $v^{i}\left[e^{\prime}\right]$ if we need to fix this $i$ th coordinate to $e$, $\bar{e}$, or some neighbor $e^{\prime} \in V\left(G_{i}\right)$ of $e$ and $\bar{e}$. Denote by $\widehat{G}_{i}$ the graph induced by $V\left(G_{i}\right) \backslash\{\bar{e}\}$ and by $G_{e}$ the subgraph of $\widehat{\Gamma}$ in which we merged the vertices of $G$ having $e$ as their $i$ th coordinate with those having $\bar{e}$. Let $\widetilde{G}_{i}$ be a star with $\bar{e}$ as central vertex and with the neighbors of $\bar{e}$ in $G_{i}$ as leaves. Let $G^{e}$ be the subgraph of $\widetilde{\Gamma}$ satisfying the following conditions: (1) $v^{i}[\bar{e}] \in V\left(G^{e}\right)$ if and only if $v^{i}[e], v^{i}[\bar{e}] \in V(G) ;(2) v^{i}\left[e^{\prime}\right] \in V\left(G^{e}\right)$ if and only if $v^{i}\left[e^{\prime}\right], v^{i}[\bar{e}], v^{i}[e] \in V(G)$, i.e., we include in $G^{e}$ the edges of $G$ which are lost by the merging operation.

Lemma 11. $\left\{\begin{array}{l}|V(G)|=\left|V\left(G_{e}\right)\right|+\left|V\left(G_{c}^{e}\right)\right| \\ |E(G)| \leq\left|E\left(G_{e}\right)\right|+\left|E\left(G_{c}^{e}\right)\right|+\left|V_{l}\left(G^{e}\right)\right| .\end{array}\right.$

Proof. The counting of vertices of $G$ is the same as in the proof of Lemma 9. The changes concern the counting of edges. The correspondence between the set $E(G)$ and the sets $E\left(G^{e}\right), E\left(G_{e}\right)$, and $V_{l}\left(G^{e}\right)$ is illustrated in Fig. 9. Namely, if $v^{i}[e], v^{i}[\bar{e}] \in V(G)$, then the edges from these vertices to their neighbors in the copy indexed by $v^{i}$ are counted once in $E\left(G_{e}\right)$ and once in $V_{l}\left(G^{e}\right)$, and the edges from these vertices to other copies are counted once in $E\left(G_{c}^{e}\right)$ and once in $E\left(G_{e}\right)$. A new edge may be created if for some $v_{1}^{i}$ and $v_{2}^{i}, v_{1}^{i}[e], v_{2}^{i}[\bar{e}] \in V(G)$ but $v_{1}^{i}[\bar{e}], v_{2}^{i}[e] \notin V(G)$. 


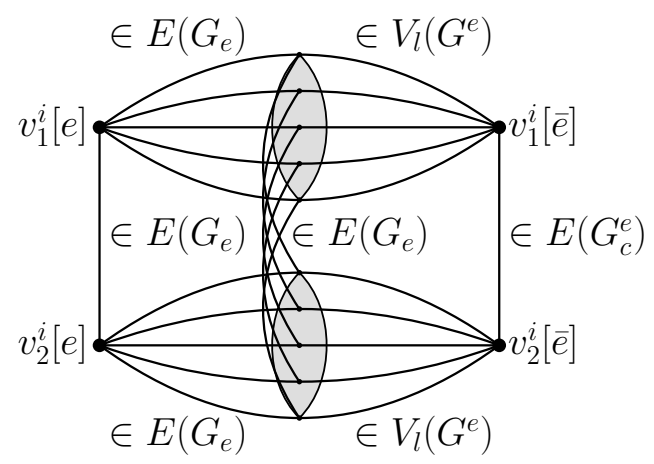

Figure 9. To the proof of Lemma 11.

With the same arguments as in Section 6.1, we can prove that $(1) \mathrm{VC}-\operatorname{dim}\left(G_{e}\right) \leq \mathrm{VC}-\operatorname{dim}(G)$ and (2) VC- $\operatorname{dim}\left(G_{c}^{e}\right) \leq \mathrm{VC}-\operatorname{dim}(G)-1$. Notice that $\left|V\left(G_{c}^{e}\right)\right|=0$ if and only if $G^{e}$ is empty. Otherwise, if a leaf $v^{i}\left[e^{\prime}\right]$ exists in $G^{e}$, the central node $v^{i}[\bar{e}]$ also exists by definition (second condition). Those leaves are the neighbors (in the copy of $\widetilde{G}_{i}$ indexed by $v^{i}$ ) of $v^{i}[\bar{e}]$ in $G^{e}$ and clearly they can not be more than $\omega(G)$, showing that $\frac{\left|V_{l}\left(G^{e}\right)\right|}{\left|V\left(G_{c}^{e}\right)\right|} \leq \omega(G)$. Finally, using Lemma 11 , the inequalities (1) and (2), and this last inequality, we obtain:

Proposition 2. $\frac{|E(G)|}{|V(G)|} \leq \omega(G) \cdot \operatorname{VC}-\operatorname{dim}(G)$.

\section{ARBoricity AND ADJACENCY LABELING SCHEMES}

An adjacency labeling scheme on a graph family $\mathcal{G}$ consists of a coding function $C_{G}: V(G) \rightarrow$ $\{0,1\}^{*}$ from the set $V(G)$ of vertices of $G$ the set $\{0,1\}^{*}$ of finite binary words that gives to every vertex of a graph $G$ of $\mathcal{G}$ a label, and a decoding function $D_{G}:\{0,1\}^{*} \times\{0,1\}^{*} \rightarrow\{0,1\}$ that, given the labels of two vertices of $G$, can determine whether they encode adjacent vertices or not. If $\mathcal{G}$ is the family of all forests on $n$ vertices, it is easy to build an adjacency labeling scheme using labels of size $2\lceil\log n\rceil$ bits. Indeed, to construct such a scheme, the coding function gives to every vertex a unique id (that requires $\lceil\log n\rceil$ bits) and concatenate to the label of each vertex the label of its parent. Given two labels, the decoding function determines if they encode adjacent vertices by testing the equality between the first half of one label and the second half of the other. It has been shown in [3] that the family of forests admits an adjacency labeling scheme using unique labels of size $\lceil\log n\rceil+O(1)$ bits.

Kannan, Naor and Rudich 32 noticed that if a graph $G$ is covered by $k$ forests, then one can build an adjacency labeling scheme with $(k+1)\lceil\log n\rceil$ bits by applying the construction mentioned above to each of the forests. The arboricity a $(G)$ of a graph $G$ is the minimal number of forests necessary to cover the edges of $G$. The classical theorem by Nash-William [36] asserts that the arboricity of a graph is almost equivalent to its density:

Theorem 4 (Nash-Williams). The edges of a graph $G=(V(G), E(G))$ can be partitioned in $k$ forests if and only if $\left|E\left(G^{\prime}\right)\right| \leq k\left(\left|V\left(G^{\prime}\right)\right|-1\right)$ for all $G^{\prime}$ subgraph of $G$. i.e., a $(G)=$ $\max _{G^{\prime} \subseteq G}\left(\frac{\left|E\left(G^{\prime}\right)\right|}{\left|V\left(G^{\prime}\right)\right|-1}\right) \leq \operatorname{dens}(G)$.

Thus, the upper bounds for the densities of graphs families provided in previous sections also bound their arboricity. We then directly obtain upper bounds on the size of labels of adjacency labeling schemes as a corollary of our results and the results of Nash-Williams [36] and Kannan and al. 32 : 
Corollary 5. Let $\Gamma:=G_{1} \square \cdots \square G_{m}$ and let $G$ be a subgraph of $\Gamma$ with $n$ vertices and induced (resp., minor) VC-dimension d (resp., $\left.d^{*}\right)$. Then, $G$ admits an adjacency labeling scheme with labels of size:

(1) $O(d \cdot \log n)$, if $\Gamma$ is an hypercube.

(2) $O\left(\beta_{0} \cdot d \cdot \log n\right)$, where $\beta_{0}=\left\lceil\max \left\{\operatorname{mad}\left(\pi_{1}(G)\right), \ldots, \operatorname{mad}\left(\pi_{m}(G)\right)\right\}\right\rceil$.

(3) $O\left(d^{*} \cdot \mu(H) \cdot \log n\right)$, if $G_{1}, \ldots, G_{m} \in \mathcal{G}(H)$.

(4) $O\left(\delta \cdot \log ^{2} n\right)$, if $G_{1}, \ldots, G_{m} \in \mathcal{G}_{\delta}$.

(5) $O\left(\log ^{k+2} n\right)$, if $G_{1}, \ldots, G_{m} \in \mathcal{G}_{\log , k}$.

(6) $O\left(\mathrm{dd}^{*}(G) \cdot d \cdot \log n\right)$, if $G_{1}, \ldots, G_{m}$ are dismantlable.

(7) $O(\omega(G) \cdot d \cdot \log n)$, if $G_{1}, \ldots, G_{m}$ are chordal graphs.

(8) $O(\omega(G) \cdot d \cdot \log n)$, if $G_{1}, \ldots, G_{m}$ are octahedra.

The assertions (1), (2), (3), (6), and (7) follow from Theorem 3 , the assertions (4) and (5) follow from Theorem 2, and the assertion (8) is a consequence of Proposition 2.

Acknowledgements. We would like to acknowledge the two referees for a careful reading of the first version and useful comments. This work was supported by ANR project DISTANCIA (ANR-17-CE40-0015).

\section{REFERENCES}

[1] I. Abraham, D. Delling, A. Fiat, A. V. Goldberg, and R. F. F. Werneck, VC-Dimension and shortest path algorithms, ICALP 2011, pp. 690-699.

[2] F. N. Afrati, C. H. Papadimitriou, and G. Papageorgiou, The complexity of cubical graphs, Inf. Contr. 66 (1985), 53-60. Corrigendum: The complexity of cubical graphs, Inf. Comput. 82 (1989), 350-353.

[3] S. Alstrup, S. Dahlgaard, and M. B. T. Knudsen, Optimal induced universal graphs and adjacency labeling for trees, FOCS 2015, pp. 1311-1326.

[4] N. Alon, and M. Tarsi, Colorings and orientations of graphs, Combinatorica 12 (1992), 125-134.

[5] M. Anthony, G. Brightwell, and C. Cooper, On the Vapnik-Chervonenkis dimension of a random graph, Discrete Math. 138 (1995), 43-56.

[6] H.-J. Bandelt and V. Chepoi, Metric graph theory and geometry: a survey, in: J. E. Goodman, J. Pach, R. Pollack (Eds.), Surveys on Discrete and Computational Geometry. Twenty Years later, Contemp. Math., vol. 453, AMS, Providence, RI, 2008, pp. 49-86.

[7] H.-J. Bandelt, V. Chepoi, A. Dress, and J. Koolen, Combinatorics of lopsided sets, Europ. J. Combin. 27 (2006), 669-689.

[8] S.L. Bezrukov, Edge isoperimetric problems on graphs, in: L. Lovász, A. Gyarfas, G.O.H. Katona, A. Recski, L. Szekely (Eds.), Graph Theory and Combinatorial Biology, Budapest 1999, in: Bolyai Soc. Math. Stud., vol.7, 1999, pp.157-197.

[9] B. Bollobás, and A.J. Radcliffe, Defect Sauer results, J. Comb. Th. Ser. A 72 (1995), 189-208.

[10] B. Brešar, J. Chalopin, V. Chepoi, M. Kovše, A. Labourel, and Y. Vaxès, Retracts of products of chordal graphs, J. Graph Theory 73 (2013), 161-180.

[11] B. Brešar, J. Chalopin, V. Chepoi, T. Gologranc, and D. Osajda, Bucolic complexes, Advances Math. 243 (2013), 127-167.

[12] J. Chalopin, V. Chepoi, H. Hirai, and D. Osajda, Weakly modular graphs and nonpositive curvature, Memoirs of Amer. Math. Soc. (to appear).

[13] M. Chastand, Fiber-complemented graphs. I, Structure and invariant subgraphs, Discrete Math. 226 (2001), no. $1-3,107-141$.

[14] N. Cesa-Bianchi and D. Haussler, A graph-theoretic generalization of Sauer-Shelah lemma, Discrete Appl. Math. 86 (1998), 27-35.

[15] V. Chepoi, B. Estellon, and Y. Vaxès, On covering planar graphs with a fixed number of balls, Discrete and Computational Geometry 37 (2007), 237-244.

[16] V. Chepoi, A. Labourel, and S. Ratel, On density of subgraphs of halved cubes, European J. Combin. (2018), doi:10.1016/j.ejc.2018.02.039.

[17] M. Deza and M. Laurent, Geometry of Cuts and Metrics, Springer-Verlag, Berlin, 1997.

[18] R. Diestel, Graph Theory, Graduate texts in mathematics, New York, Berlin, Paris, Springer, 1997.

[19] D.Ž. Djoković, Distance-preserving subgraphs of hypercubes, J. Combin. Th. Ser. B 14 (1973), $263-267$. 
[20] M.R. Garey and R.L. Graham, On cubical graphs, J. Combin. Th. B 18 (1975), 84-95.

[21] R.L. Graham, On primitive graphs and optimal vertex assignments, Ann. N. Y. Acad. Sci. 175 (1970), 170-186.

[22] R.L. Graham and P.M. Winkler, On isometric embeddings of graphs, Trans. Amer. Math. Soc. 288 (1985), 527-536.

[23] R. Hammack, W. Imrich, and S. Klavžar, Handbook of Product Graphs, Second Edition, CRC Press, Boca Raton, 2011.

[24] L.H. Harper, Optimal assignments of numbers to vertices, SIAM J. Appl. Math. 12 (1964), 131-135.

[25] L.H. Harper, Global Methods for Combinatorial Isoperimetric Problems, Cambridge Studies in Advanced Mathematics (No. 90), Cambridge University Press 2004.

[26] D. Haussler, Sphere packing numbers for subsets of the Boolean $n$-cube with bounded Vapnik-Chervonenkis dimension, J. Comb. Th. Ser. A 69 (1995), 217-232.

[27] D. Haussler, N. Littlestone, and M. K. Warmuth, Predicting $\{0,1\}$-functions on randomly drawn points, Inf. Comput. 115 (1994), 248-292.

[28] D. Haussler and P.M. Long, A generalization of Sauer's lemma, J. Combin. Th. Ser. A 71 (1995), 219-240.

[29] D. Haussler and E. Welzl, $\epsilon$-Nets and simplex range queries, Discrete and Computational Geometry 2 (1987), 127-151.

[30] P. Hell and J. Nešetřil, Graphs and Homomorphisms, New York, Oxford University Press, 2004.

[31] Z. Füredi and J. Pach, Traces of finite sets: extremal problems and geometric applications, Extremal Problems for Finite Sets (Visegrád, Hungary, 1991), pp. 251-282, Bolyai Society Mathematical Studies, 3.

[32] S. Kannan, M. Naor, and S. Rudich, Implicit representation of graphs, SIAM J. Discr. Math. 5 (1992), 596-603.

[33] E. Kranakis, D. Krizanc, B. Ruf, J. Urrutia, and G. Woeginger, The VC-dimension of set systems defined by graphs, Discrete Appl. Math. 77 (1997), 237-257.

[34] D. Kuzmin and M.K. Warmuth, Unlabelled compression schemes for maximum classes, J. Mach. Learn. Res. 8 (2007), 2047-2081.

[35] J. Lawrence, Lopsided sets and orthant-intersection of convex sets, Pacific J. Math. 104 (1983), $155-173$.

[36] C. St J.A. Nash-Williams, Edge-disjoint spanning trees of finite graphs, J. London Math. Soc. 1 (1961), 445-450.

[37] B. K. Natarajan, On learning sets and functions, Machine Learning 4 (1989), 67-97.

[38] R. Nowakowski and P. Winkler, Vertex-to-vertex pursuit in a graph, Discrete Math. 43 (1983), $235-239$.

[39] D. Pollard, Convergence of Stochastic Processes, Springer Science \& Business Media, 2012.

[40] B.I. Rubinstein, P.L. Bartlett, and J.H. Rubinstein, Shifting: one-inclusion mistake bounds and sample compression, J. Comput. Syst. Sci. 75 (2009), 37-59.

[41] N. Sauer, On the density of families of sets, J. Combin. Th. Ser. A 13 (1972), 145-147.

[42] S.V. Shpectorov, On scale embeddings of graphs into hypercubes, Europ. J. Combin. 14 (1993), $117-130$.

[43] V.N. Vapnik and A.Y. Chervonenkis, On the uniform convergence of relative frequencies of events to their probabilities, Theory Probab. Appl. 16 (1971), 264-280. 